Computing and Informatics, Vol. 39, 2020, 264 297, doi: 10.31577/cai_2020_1-2 264

\title{
A LOGIC PETRI NET-BASED REPAIR METHOD OF PROCESS MODELS WITH INCOMPLETE CHOICE AND CONCURRENT STRUCTURES
}

\author{
Yuanxiu Teng, Liang QI*, Yuyue DU \\ The College of Computer Science and Engineering \\ Shandong University of Science and Technology, Qingdao 266590, China \\ e-mail: 392828580@qq.com, \{qiliangsdkd, yydu001\}@163.com
}

\begin{abstract}
Current model repair methods cannot repair incomplete choice and concurrent structures precisely and simply. This paper presents a repair method of process models with incomplete choice and concurrent structures via logic Petri nets. The relation sets are constructed based on process trees, including branch sets, choice activity sets and concurrent activity sets. The deviations are determined by analyzing the relation between relation sets and activities in the optimal alignment. The model repair method is proposed for models with incomplete choice and concurrent structures via logic Petri nets according to different deviation positions. Finally, the correctness and effectiveness of the logic Petri net-based repair method are illustrated by simulation experiments.
\end{abstract}

Keywords: Process model, model repair, process tree, alignment, logic Petri net

\section{INTRODUCTION}

Process mining builds a bridge between data mining and process modeling and analysis. It extracts effective information from the data and resources of the real business process system, and builds the process model based on different algorithms according to the required information. Process mining is widely used in the design, analysis, implementation and adjustment of the system process [1]. The three types of applications for process mining is process discovery, conformance checking, and process improvement. The process discovery algorithm is a function that maps the

* Corresponding author 
event log to a process model, which can be a BPMN [2], YAWL [3], Petri net [4, 5] and so on. $\alpha$ algorithm takes an event $\log$ as the input, finds out the possible causal dependence according to the sequence of activities, and outputs a Petri net with the initial identification [6. Heuristic mining builds models with the use of representation preference and frequency of causal networks. The basic idea of heuristic mining algorithm is that infrequent paths should not be included in the model [7]. Conformance checking is used to compare the behaviors of process models with the behaviors recorded in the event logs, and it looks for their commonality and heterogeneity, so as to ensure that the information system and the actual business process keep a good compliance. The classic conformance checking contains token replay and alignments [8]. Besides, the method proposed by [9] projects both systems and system models or logs onto sub-sets of activities to determine their performance, and is applicable to both log-model and model-model conformance checking. The literature [10] can analyze and classify deviations with respect to the intended purpose of data, and provides an algorithm to identify wide range of deviations.

Conformance checking can also be used to improve business processes, repair models, and evaluate process discovery algorithms [11. When the process model and event logs do not match on the process, the process model needs to be repaired, which is the process improvement. The aim of process improvement is to make the process model better reflect the real business system and improve the performance of the model. Fitness, simplicity, precision, and generalization are four main types of model performance, and those four performances are used to evaluate the quality of process models. A new genetic process mining algorithm is proposed to discover a process model from event logs, and it uses the tree representation to ensure the soundness of the model [12. To improve the quality of process models, many approaches of process improvement are proposed. The Fahland's approach uses alignments to align the runs of the given process models to the traces in the logs 13 . It mines loops that can replay sub-logs of non-fitting sub-traces. The Goldratt's approach and Knapsack's approach are proposed to improve the correspondence between a model and event logs, and speed up the repair [14]. The work in [15] presents a judgment to mine the sub-process as the branch of choice structures, instead of inserting the sub-process directly into the original model.

Logic Petri net is the further abstraction and extension of the Petri net with inhibitor arcs [16, 17]. It is more concise and can describe a large number of logic relations among complex activities. From the perspective of analyzing business process operation and resources, logic Petri nets can better analyze batch processing function and the uncertainty of activity enablement of business process systems. The work in [16] proposes a vector matching method, and analyzes the reachability, liveness, conservativeness, and reversibility of logic Petri nets based on reachability trees and the state equations. The precursor and successor of activities in the traces are defined in [17, and an extended log-based ordering relationship is proposed. The work in [18] is based on alignments to repair unfitting transitions in concurrent blocks and generates a new branch containing new activities. In some real business processes, concurrency and choice exist at the same time, we call this structure is an 
incomplete choice structure or incomplete concurrent structure. The current model repair methods based on Petri nets consider to improve the fitness of models, often ignore the precison and simplicity, and cannot correctly describe the logic relation among activities.

Therefore, we present a logic Petri net-based repair method. This work has the following contributions:

1. Relation sets are constructed based on process trees, including choice and concurrent activity sets, and branch sets. These relation sets can precisely locate deviations for concurrent and choice structures combining with optimal alignments.

2. The algorithms of determining deviations are presented based on relation sets and event logs. The model repair method is proposed for models with incomplete choice and concurrent structures via logic Petri nets.

3. Experimental results illustrate the correctness and effectiveness of the repair method presented in the paper.

The rest of the paper is organized as follows. The background in relevant fields is introduced in Section 2. Section 3 presents an approach to repair models with incomplete choice structures. The method of repairing models with incomplete concurrent structures is proposed in Section 4. The results and performance analysis of simulation experiments are given in Section 5. Section 6 concludes our work and draws the future work.

\section{PRELIMINARIES}

This section introduces some basic notions, mainly including Petri nets [4, logic Petri nets [16, alignments [11], process trees [14, and the precursor and successor [17]. In the following content, $\mathcal{N}$ represents a natural number set, i.e., $\mathcal{N}=\{0,1,2, \ldots\}$.

Definition 1 (Trace, event $\log$ ). Let $A \subseteq \mathcal{A}$ be a set, and $\mathcal{A}$ is all sets of activities. $\sigma \in A^{*}$ is called a trace that denotes a sequence of activities. An event $\log$ is a multi-set of traces denoted as $L \in \mathcal{B}\left(A^{*}\right)$.

Definition 2 (Tuple). Let $A$ be a set and a tuple with $n$ elements is denoted by $r=\left(b_{1}, b_{2}, \ldots, b_{n}\right) \in A \times A \times \cdots \times A$. The $i^{\text {th }}$ element of $r$ is denoted as $\pi_{i}(r)$.

For example, there is a tuple $r=(x, y, z) \in A \times A \times A$ with 3 elements, $\pi_{1}(r)=x$, $\pi_{2}(r)=y$, and $\pi_{3}(r)=z$.

Definition 3 (Pre-set, post-set). Let $N=(P, T ; F)$ be a net. $P$ denotes a finite set of places, $T$ denotes a finite set of transitions. $F \subseteq(P \times T) \cup(T \times P)$ denotes a finite set of directed arcs with each one from $p$ to $t$ or from $t$ to $p$, where $p \in P$ 
and $t \in T . x$ is a node in $N$ and $\forall x \in P \cup T$, we have

$$
\begin{aligned}
& \bullet x=\{y \mid y \in(P \cup T) \wedge(y, x) \in F\}, \\
& x^{\bullet}=\{y \mid y \in(P \cup T) \wedge(x, y) \in F\}
\end{aligned}
$$

where ${ }^{\bullet} x$ and $x^{\bullet}$ represent the pre-set and post-set of $x$, respectively.

Definition 4 (Petri net). A four-tuple $P N=(P, T ; F, M)$ is a Petri net, where

1. $N=(P, T ; F)$ is a net;

2. $M: P \rightarrow \mathcal{N}$ is a marking, $M(p)$ denotes the number of tokens in $p$, where $p \in P$; and

3. $P N$ has the following transition firing rules:

(a) for $t \in T$, if $\forall p \in \in^{\bullet} t: M(p) \geq 1$, then $t$ is enabled under $M$, denoted as $M[t>$; and

(b) if $M\left[t>\right.$, then $t$ can fire, and a new marking $M^{\prime}$ is generated, denoted as $M\left[t>M^{\prime}\right.$, and for $\forall p \in P$, we have

$$
M(P)^{\prime}= \begin{cases}M(P)-1, & p \in \in^{\bullet} t t^{\bullet} \\ M(P)+1, & p \in t^{\bullet} \bullet t \\ M(P), & \text { otherwise }\end{cases}
$$

Definition 5 (Logic Petri net). A six-tuple $L P N=(P, T ; F, I, O, M)$ is called a logic Petri net, where

1. $P$ denotes a finite set of places;

2. $T=T_{D} \cup T_{I} \cup T_{O}$ denotes a finite set of transitions, and $T \cap P=\phi$, for $\forall t \in T$, $\bullet t \cap t^{\bullet}=\phi$, where

(a) $T_{D}$ is a set of classic transitions as in a Petri net;

(b) $T_{I}$ is a set of logic input transitions, for $\forall t \in T_{I}$, the input place of $t$ is restricted by a logic input function $f_{I}(t)$; and

(c) $T_{O}$ is a set of logic output transitions, for $\forall t \in T_{O}$, the output place of $t$ is restricted by a logic output function $f_{O}(t)$;

3. $F \subseteq(P \times T) \cup(T \times P)$ denotes a set of directed arcs with each one from $p$ to $t$ or from $t$ to $p$, where $p \in P$ and $t \in T$;

4. $I$ denotes a logic input function of $t$, where $t \in T_{I}$, and for $\forall t \in T_{I}, I(t)=f_{I}(t)$;

5. $O$ denotes a logic output function of $t$, where $t \in T_{O}$, and for $\forall t \in T_{O}, O(t)=$ $f_{O}(t)$

6. $M: P \rightarrow \mathcal{N}$ is a marking, $M(p)$ denotes the number of tokens in $p$, where $p \in P$; and

7. $L P N$ has the following transition firing rules: 
(a) for $\forall t \in T_{D}$, the transition firing rule is consistent with that of Petri nets;

(b) for $\forall t \in T_{I}, I(t)=f_{I}(t)$. If $\left.f_{I}(t)\right|_{M}=T_{\bullet} T_{\bullet}$, then $t$ can fire and is denoted as $M\left[t>M^{\prime}\right.$, and for $\forall p \in \in^{\bullet} t, M(p)=1, M^{\prime}(p)=0$; for $\forall p \in t^{\bullet}, M(p)=0$, $M^{\prime}(p)=1$; and for $\forall p \notin^{\bullet} t \cup t^{\bullet}, M^{\prime}(p)=M(p)$; and

(c) for $\forall t \in T_{O}, O(t)=f_{O}(t)$. If $\left.f_{O}(t)\right|_{M}=$. $_{\bullet}$, then $t$ can fire and is denoted as $M\left[t>M^{\prime}\right.$, and $\forall p \in \in^{\bullet} t, M^{\prime}(p)=0$; for $\forall p \in t^{\bullet}, M^{\prime}(p)=1$; and for $\forall p \notin^{\bullet} t \cup t^{\bullet}, M^{\prime}(p)=M(p)$.

8. There are three symbols of the logic function: $\otimes, \wedge$ and $\vee . p_{1} \otimes p_{2} \cdots \otimes p_{n}$ denotes only one of $p_{1}-p_{n}$ contains tokens; $p_{1} \wedge p_{2} \cdots \wedge p_{n}$ denotes each of $p_{1}-p_{n}$ contains tokens; $p_{1} \vee p_{2} \cdots \vee p_{n}$ denotes at least one of $p_{1}-p_{n}$ contains tokens; where $n \geq 2$.

For example, a logic Petri net denoted by $L P N_{1}$ is presented in Figure 1, where $t_{1}$ and $t_{3}$ are two logic transitions and $t_{2}$ is a classic transition. $t_{1}$ is a logic input transition, and $I\left(t_{1}\right)=p_{2} \wedge\left(p_{1} \otimes p_{3}\right)$. If $t_{1}$ fires, $p_{1}$ and $p_{3}$ cannot contain tokens at the same time, and $f_{I}\left(t_{1}\right)=p_{2} \wedge\left(p_{1} \otimes p_{3}\right)=\bullet T_{\bullet}$, there will be two cases:

1. both $p_{1}$ and $p_{2}$ contain a token; or

2. both $p_{2}$ and $p_{3}$ contain a token.

Besides, $t_{3}$ is a logic output transition, and $O\left(t_{3}\right)=p_{6} \vee p_{7}$. When $t_{3}$ fires, its logic output function needs to satisfy $f_{O}\left(t_{3}\right)=p_{6} \vee p_{7}=\bullet T_{\bullet}$, there are three cases:

1. only $p_{6}$ contains a token;

2. only $p_{7}$ contains a token; or

3. both $p_{6}$ and $p_{7}$ contain a token.

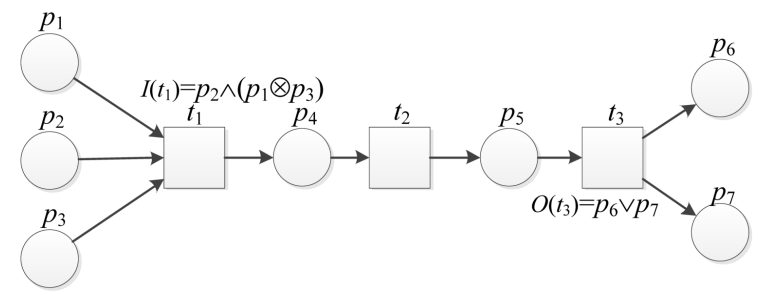

Figure 1. A logic Petri net model $L P N_{1}$

Definition 6 (Alignment). Let $\sigma \in A^{*}$, and $P N=(P, T ; F, M)$. A move is a pair $(a, t) \in(A \cup>>) \times(T \cup>>)$, where $>>$ denotes no move. A move sequence denoted by $\gamma=\left(\left(a_{1}, t_{1}\right)\left(a_{2}, t_{2}\right) \ldots\left(a_{|\gamma|}, t_{|\gamma|}\right)\right)$ is called an alignment between $\sigma$ and $P N$, where

1. $\pi_{1}(\gamma)=\sigma$ denotes a trace sequence generated by $\gamma$ (ignoring $>>$ ); and 
2. $m_{i}\left[\pi_{2}(\gamma)>m_{f}\right.$ denotes a complete firing sequence generated by $\gamma$ (ignoring $>>$ ); and

3. for each move $(a, t)$, it is called a log activity if $a \in A$ and $t=>>$; it is called a model activity if $a=>>$ and $t \in T$; it is called a synchronous activity if $a \in A$ and $t \in T$; it is called an illegal activity otherwise.

$\Gamma_{\sigma, P N}$ denotes a set of all alignments between $\sigma$ and $P N$.

Definition 7 (Optimal alignment). Let $\sigma \in A^{*}$ and $P N=(P, T ; F, M) \cdot \gamma^{\prime} \in \Gamma_{\sigma, P N}$ denotes an optimal alignment between $\sigma$ and $P N$, if for $\forall \gamma^{\prime} \in \Gamma_{\sigma, P N}$, we have $\sum_{(a, t) \in \gamma} l c(a, t) \leq \sum_{\left(a^{\prime}, t^{\prime}\right) \in \gamma} l c\left(a^{\prime}, t^{\prime}\right)$, where $l c(a, t)$ denotes a likelihood cost function. For each move $(a, t)$, if $a \in A$ and $t=>>, l c(a, t)=1$; if $a=>>$ and $t \in T$, $l c(a, t)=1$; if $a \in A$ and $t \in T, l c(a, t)=0 . \Gamma_{\sigma, P N, l c}$ denotes a set of all optimal alignments between $\sigma$ and $P N$.

Definition 8 (Process tree). Let $A \in \mathcal{A}$ be a set of activities. The notation of $\bigoplus$ denotes an operator set, and $\bigoplus=\{\times, \rightarrow, \odot, \wedge\}$, where

1. $a \in A \cup\{\tau\}$ denotes a process tree, and $\tau$ is an invisible transition; and

2. if $P T_{1}, P T_{2}, \ldots, P T_{n}(n>0)$ are process trees, and then $\bigoplus\left(P T_{1}, \ldots, P T_{n}\right)$ is also a process tree; $\times$ represents the choice relation among $P T_{1}, \ldots, P T_{n} ; \rightarrow$ represents the sequential execution of $P T_{1}, \ldots, P T_{n} ; \bigodot$ represents the loop structure of $P T_{1}, \ldots, P T_{n}$; and $\wedge$ represents the parallel structure of $P T_{1}, \ldots, P T_{n}$.

Definition 9 (Precursor, successor). Let $L \in B\left(A^{*}\right)$ be a $\log$ where $A \in \mathcal{A}$. For $\forall \sigma \in L$, if an activity $a \in \delta(\sigma)$ and the position index of $a$ in $\sigma$ is $i$, the precursor of $a$ is denoted as $\triangle_{a}$ at the position with index $i-1$; and the successor of $a$ is denoted as $a^{\triangle}$ at the position with index $i+1$.

For example, there is a trace $\left\langle t_{1}, t_{2}, t_{5}, t_{3}, t_{4}, t_{5}\right\rangle, t_{5}$ is the precursor of $t_{3}$, i.e., $\triangle_{t_{3}}=t_{5} ; t_{4}$ is the successor of $t_{3}$, i.e., $t_{3}^{\triangle}=t_{4}$.

\section{INCOMPLETE CHOICE STRUCTURES DEVIATION REPAIR}

This section proposes a repair method of process models with incomplete choice structures. For incomplete choice structures, we first present choice relation sets based on process trees of process models, including head-to-tail places, branch sets and choice activity sets. By comparing and analyzing the logic relation between activities in traces and transitions of choice relation sets, a model repair method via logic Petri nets is proposed.

In the following, we use $P N=(P, T ; F, M)$ to denote a four-tuple Petri net, and use $P T$ to denote a process tree of $P N$.

Definition 10 (Tree relation). Let $\mid \rightarrow$ be a relation symbol of PT, and $\left(t_{i} \cup\right.$ $\bigoplus)^{*}\left|\rightarrow\left(t_{j} \cup \bigoplus\right)^{*}\right| \rightarrow \ldots \mid \rightarrow\left(t_{n} \cup \bigoplus\right)^{*}$ is a tree relation, where $t_{i}, t_{j}, \ldots, t_{n} \in T$ and $\bigoplus=\{\times, \rightarrow, \odot, \wedge\}$. 
Definition 11 (Node relation). Let $n \in(T \cup \bigoplus)$ be a node of $P T, \bigoplus=\{\times, \rightarrow$, $\odot, \wedge\}$. The top layer of $P T$ is called the root node, there are six node relation:

1. if $m \mid \rightarrow n$ and $m \in(T \cup \bigoplus)$, then $m$ is called the parent node of $n$, denoted as $n$.parent $=m$;

2. if $n \mid \rightarrow m$ and $m \in(T \cup \bigoplus)$, then $m$ is called the child node of $n$, denoted as n. child $=m$;

3. if $m|\rightarrow n, m| \rightarrow l, m \mid \rightarrow r$ and $m, l, r \in(T \cup \bigoplus)$, then $l$ and $r$ are called the left and right sibling nodes of $n$, respectively, where $l$ and $r$ are on the left and right sides of $n$, denoted as $n . l s i b=l$ and $n . r s i b=r$;

4. if $\exists$ n.child $\in(T \cup \bigoplus)$ and $\mid$ n.child $\mid>1$, then n.child. $p_{i}$ denotes the $i^{\text {th }}$ child node of $n$, where $i \in[1, \mid$ n.child $\mid]$;

5. if $n|\rightarrow \ldots| \rightarrow m$, m. child $=$ null, and $m$.lib $=$ null, then $m$ is called the leftmost leaf node of $n$, denoted as n.child.lp $=m$; and

6. if $n|\rightarrow \ldots| \rightarrow m$, m.child $=$ null, and $m$.rib $=$ null, then $m$ is called the rightmost leaf node of $n$, denoted as n.child.rp $=m$.

For example, a Petri net model denoted by $P N_{1}$ is presented in Figure 2, and $P N_{1}$ contains choice structures. Let $\sigma_{1}=\left\langle a, t_{1}, t_{5}, t_{2}, t_{4}, b\right\rangle, \sigma_{2}=\left\langle a, t_{6}, t_{7}, c, b\right\rangle$ be two traces. $P N_{1}$ has a choice structure with transitions $t_{1}, t_{2}, t_{3}, t_{4}, t_{5}, t_{6}, t_{7}$. In the trace $\sigma_{1}$, we have that $t_{1}, t_{2}, t_{4}$ and $t_{5}$ are concurrently enabled. It shows that the branch containing $t_{1}, t_{2}, t_{4}$ can occur concurrently with the branch containing $t_{5}$. In the original model $P N_{1}$, either of these two branches can be selected to occur. So there is an incomplete choice structure.

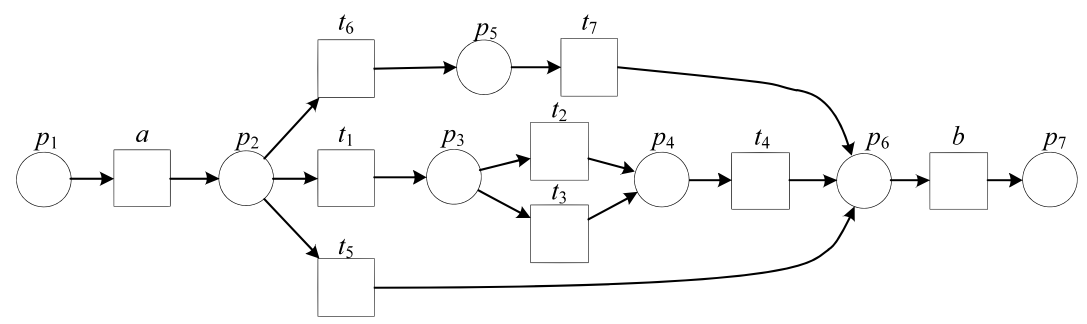

Figure 2. A Petri net model $P N_{1}$

Figure 3 shows the process tree of $P N_{1}$ represented by $P T_{1} . P T_{1}=\rightarrow(a, \times((\rightarrow$ $\left.\left.\left(t_{1}, \times\left(t_{2}, t_{3}\right), t_{4}\right), t_{5}, \rightarrow\left(t_{6}, t_{7}\right)\right), b\right)$. For $P T_{1}, " \rightarrow " \mid \rightarrow$ " $\times ", " \times " \mid \rightarrow " \rightarrow "$, and $" \rightarrow " \mid \rightarrow t_{1}$ are three tree relations of $P T_{1}$. The root node is " $\rightarrow$ ". If $n=$ " $\times "$, then n.parent $=" \rightarrow "$ ", n.lsib $=a$, and n.rsib $=b$. It can be seen that $t_{5}$ is the child node of $n$, n.child. $p_{1}=" \rightarrow$ ", n.child. $p_{2}=t_{5}$, and n.child. $p_{3}=" \rightarrow$ ". Besides, n.child.lp $=t_{1}$, and n.child.rp $=t_{7}$.

Definition 12 (Head-to-tail place). $\left[S F_{P}, S L_{P}\right]$ is called the head-to-tail place of a choice structure, where $S F_{P}$ and $S L_{P}$ are called the head place and the tail place, respectively. Let $n=$ " $\times$ " be a node of $P T$, and it needs to satisfy: 


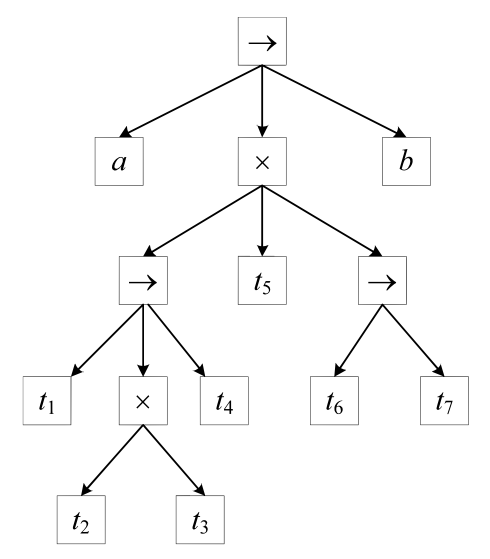

Figure 3. The process tree $P T_{1}$ of $P N_{1}$

1. if n.child. $p_{i}=m$, m.child $=$ null, and $0<i \leq \mid$ n.child $\mid$, then $S F_{P}=\bullet^{\bullet} m$ and $S L_{P}=m^{\bullet} ;$ and

2. if n.child. $p_{i}=m, \exists$ m.child $\in(T \cup \bigoplus), \bigoplus=\{\times, \rightarrow, \odot, \wedge\}$ and $0<i \leq \mid$ n.child $\mid$, then $S F_{P}=\bullet$ (n.child.lp) and $S L_{P}=(\text { n.child.rp })^{\bullet}$.

Definition 13 (Branch set). Let $n$ be a node of PT, $n=" \times "$ or $n=" \wedge "$, and a branch is defined as follows:

1. If $n$. child $=m$, m.child $=$ null, i.e., n.child.child $=$ null, then $\{m\}$ is a branch; and

2. if n.child $=m, \exists$ m.child. $p_{1}$, m.child. $p_{2}, \ldots$, m.child. $p_{k}$, and $0<k \leq \mid$ m.child $\mid$, where

(a) if $m=$ " $\rightarrow$ ", then $\left\{\right.$ m.child. $p_{1}$, m.child. $p_{2}, \ldots$, m.child. $\left.p_{k}\right\}$ is a branch;

(b) if $m=$ " $\times$ ", then $\left\{\right.$ m.child. $\left.p_{1}\right\},\left\{\right.$ m.child. $\left.p_{2}\right\}, \ldots,\left\{\right.$ m.child. $\left.p_{k}\right\}$ are each a branch; and

(c) if $m=" \wedge "$, then $\left\{\right.$ m.child. $\left.p_{1}\right\},\left\{\right.$ m.child. $\left.p_{2}\right\}, \ldots,\left\{\right.$ m.child. $\left.p_{k}\right\}$ are each a branch.

$B_{S}$ denotes a branch set that contains all branches of choice and concurrent structures.

Theorem 1. For $n=$ " $\times$ ", if $\exists$ n.child $=m$ and m.child $=$ null, then $\{m\}$ is a branch.

Proof. If $\exists$ n.child $=m$ and $m$.child $=$ null, i.e., $\exists$ n.child.child $=$ null, it means that the child node of $n$ is a leaf node. $\neg \exists$ m.child $\rightarrow \bigoplus$, where $\bigoplus=\{\times, \rightarrow, \odot, \wedge\}$, i.e., there are no structures behind $m$ until $S L_{P}$. We have $S L_{P}=m \bullet$. Since $m$.parent $=n$ and $n=$ " $\times$ ", $n$ is the initial operator notation of the choice structure, then $S F_{P}=\bullet$ $m$. Thus, $\{m\}$ is a branch. 
If $\{m\}$ is a branch and $n=$ " $\times$ ", it means $m^{\bullet}=S L_{P}$ and $\bullet=S F_{P}$. We have $\neg \exists$ m.child $\mid \rightarrow \bigoplus$ and " $\times " \mid \rightarrow m$, i.e., $m=$ n.child and $m$.child $=$ null.

Theorem 1 indicates that for the node of the process tree, if the node is " $\times$ " and the child node of the node is a leaf node, then the child node alone is a branch of the choice structure.

Theorem 2. For $n=" \times "$ or $n=" \wedge ", m=$ n.child, $0<k \leq \mid$ m.child $\mid$, $\left\{\right.$ m.child. $\left.p_{1}\right\},\left\{\right.$ m.child. $\left.p_{2}\right\}, \ldots,\left\{\right.$ m.child. $\left.p_{k}\right\}$ are each a branch.

Proof. For $n=" \times "$ or $n=" \wedge$ ", there is $\exists q=$ m.lsib or $\exists q=m$.rsib, and $n \mid \rightarrow$ m.lsib or $n \mid \rightarrow$ m.rsib. If q.child.lp and $m$ are contained in a branch, we have $M\left[\right.$ q.child.lp $>, \neg M\left[m>, M\left[\right.\right.$ q.child.lp $>M^{\prime}, M^{\prime}[m>$ or $M[m>, \neg M[$ q.child.lp $>$, $M\left[m>M^{\prime}, M^{\prime}[\right.$ q.child.lp $>$. For $n=$ " $\times ", \exists M, M[$ q.child.lp >, $\neg M[m>$, when $M\left[\right.$ q.child.lp > $M^{\prime}, \neg M^{\prime}\left[m>\right.$ or $M\left[m>, \neg M\left[\right.\right.$ q.child.lp >, $M\left[m>M^{\prime}\right.$, $\neg M^{\prime}[$ q.child.lp $>$, i.e., q.child.lp and $m$ are not contained in a branch. For $n=$ "^", $\exists M, M\left[\right.$ q.child.lp >, $M\left[m>, M\left[\right.\right.$ q.child.lp $>M^{\prime}, M^{\prime}\left[m>\right.$ or $M\left[m>M^{\prime}\right.$, $M^{\prime}[$ q.child.lp $>$, i.e., q.child.lp and $m$ are not contained in a branch. Thus, $\left\{\right.$ m.child. $\left.p_{1}\right\},\left\{\right.$ m.child. $\left.p_{2}\right\}, \ldots,\left\{\right.$ m.child. $\left.p_{k}\right\}$ are each a branch.

By Theorem 2, for the node denoted by " $\times$ " or " $\wedge$ " of the process tree, those child nodes of this node are not in the same branch.

Definition 14 (Choice activity set). The choice activity set contains first and last choice activity sets. $F C_{S}$ and $L C_{S}$ denote the first and the last choice activity sets, respectively. If $n=$ " $\times$ " is a node of $P T$, we have:

1. if $n$.child $=m$, m. child $=$ null, then $F C_{S}=\{m\}$, and $L C_{S}=\{m\}$;

2. if $n|\rightarrow \ldots| \rightarrow m, m=$ " $\wedge$ " or $m=$ " $\times ", k=\mid$ m.child $\mid, q=$ n.child.lp and $m=$ q.parent, then $F C_{S}=\left\{\right.$ m.child. $p_{1}$, m.child. $p_{2}, \ldots$, m.child. $\left.p_{k}\right\}$;

3. if $n|\rightarrow \ldots| \rightarrow m, m=$ " $\wedge$ " or $m=$ " $\times ", k=\mid$ m.child $\mid, q=$ n.child.rp and $m=q$. arent, then $L C_{S}=\left\{\right.$ m.child. $p_{1}$, m.child. $p_{2}, \ldots, m$. child. $\left.p_{k}\right\} ;$ and

4. if $n|\rightarrow \ldots| \rightarrow m, m=$ " $\rightarrow$ ", and m.child.child $=$ null, then $F C_{S}=\{$ m.child.lp $\}$, and $L C_{S}=\{$ m.child.rp $\}$.

The algorithms of calculating branch sets, head-to-tail place and choice activity sets are proposed in the following.

Algorithm 1 calculates the branch set. For each node $n$ of the process tree, if $n$ is " $\wedge$ " or " $\times$ ", and the child node of $n$ is a leaf node, then this child node of $n$ is contained in a branch. If the child node of $n$ is " $\rightarrow$ ", and those child nodes of " $\rightarrow$ " are leaf nodes, then all those leaf nodes are contained in a branch. If the child node of " $\rightarrow$ " is " $x$ ", then each node of the child node of " $x$ " is contained in a branch.

Algorithm 2 calculates the head-to-tail place and the choice activity set. For each node $n$ of the process tree, if $n$ is " $\times$ ", its head place is the pre-set of the leftmost leaf node of $n$, and its tail place is the post-set of the rightmost leaf node 


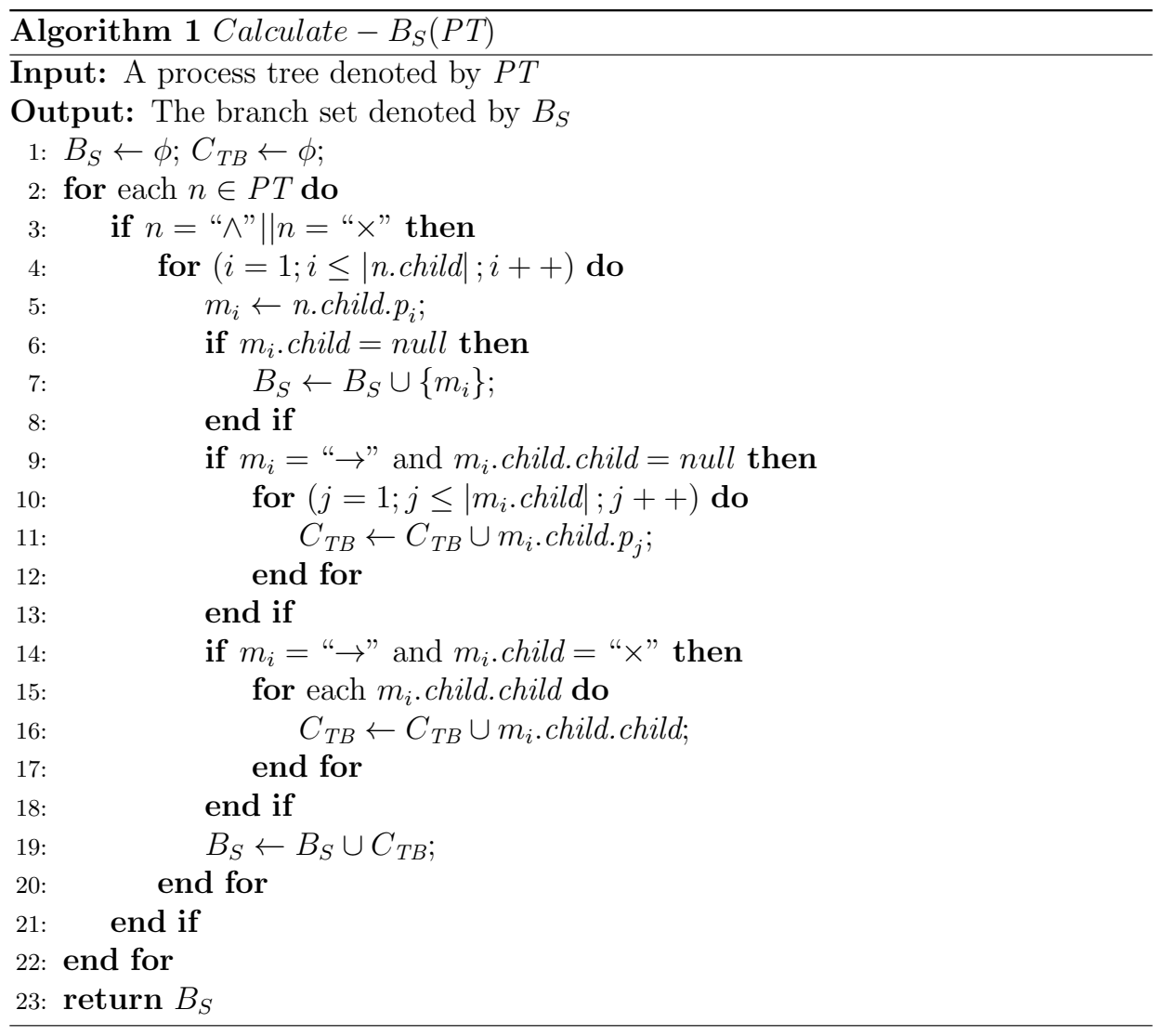

of $n$. If the child node is a leaf node, then it is both contained in the first and last choice activity set. If the child node $g$ of $n$ is " $\times$ " or " $\wedge$ ", and $g$ is the parent node of the leftmost of rightmost leaf node of $n$, then each child node of $g$ is contained in the first or last choice activity set. If $g$ is " $\rightarrow$ " and the child node of $g$ is a leaf node, then the leftmost leaf node of $g$ in contained in the first choice activity set, and the rightmost leaf node of $g$ in contained in the last choice activity set.

For $P T_{1}$, we can obtain its head-to-tail place, branch set and choice activity set. Its head-to-tail place is denoted by $\left[S F_{P}, S L_{P}\right]=\left[p_{2}, p_{6}\right]$, and its branch set is denoted by $B_{S}=\left\{\left\{t_{1}, t_{2}, t_{4}\right\},\left\{t_{1}, t_{3}, t_{4}\right\},\left\{t_{5}\right\},\left\{t_{6}, t_{7}\right\}\right\}$. The first choice activity set is denoted by $F C_{S}=\left\{t_{1}, t_{5}, t_{6}\right\}$ and the last choice activity set is denoted by $L C_{S}=\left\{t_{4}, t_{5}, t_{7}\right\}$.

Theorem 3. $\left|B_{S}\right|_{\max }$ is the maximum length of elements in $B_{S}$. For $n=$ " $\times$ " or $n=" \wedge ", n|\rightarrow w| \rightarrow \ldots \mid \rightarrow m$, if $w=" \rightarrow ", \exists m=" \rightarrow ", x=\mid$ w.child $\mid$ and $y=$ $\mid$ m.child $\mid$, then $\left|B_{S}\right|_{\max }=x+y$, where w.child.child $=$ null and m.child.child $=$ null; If $\neg \exists m=" \rightarrow ",\left|B_{S}\right|_{\max }=x+1$. 


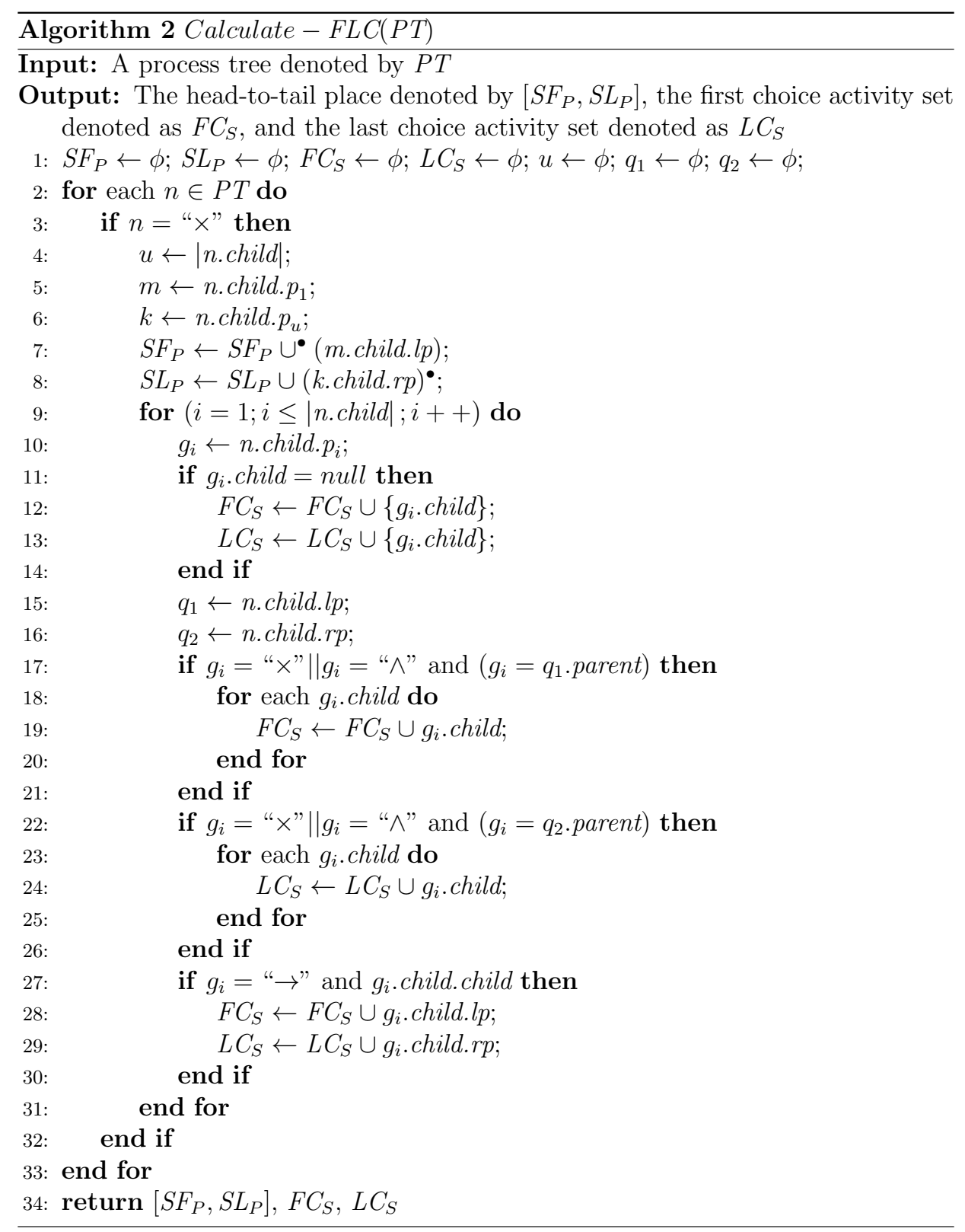


Proof. For $n=$ "×" or $n=" \wedge ", n|\rightarrow w| \rightarrow \ldots \mid \rightarrow m, w=" \rightarrow$ ", if $\exists d_{1}=$ w.child. $p_{i}, d_{1}$.child $=$ null, $d_{2}=$ w.child. $p_{j}, d_{2}$.child $=$ null, where $i, j \in[1, \mid$ w.child $\mid]$, it means that $M\left[d_{1}>, \neg M\left[d_{2}>M^{\prime}, M\left[d_{1}>M^{\prime}, M^{\prime}\left[d_{2}>\right.\right.\right.\right.$, so the maximum length of the element of $B_{S}$ only containing $d_{1}$ and $d_{2}$ is 2 . So if $\exists d=w$.child, d.child $=$ null, $|d|=x$, where $0<x \leq \mid$ w.child $\mid$, then the length of the element in $B_{S}$ containing $d$ is $x$. If $\exists m=$ " $\rightarrow$ ", if $\exists e_{1}=m$.child. $p_{i}, e_{1}$.child $=$ null, $e_{2}=$ m.child. $p_{j}, e_{2}$. child $=$ null, where $i, j \in[1, \mid m$.child $\mid]$, it means that $M\left[e_{1}>, \neg M\left[e_{2}>M^{\prime}, M\left[e_{1}>M^{\prime}\right.\right.\right.$, $M^{\prime}\left[e_{2}>\right.$, in the same way, the length of the element in $B_{S}$ only containing $e_{1}$ and $e_{2}$ is 2. So if $\exists e=m$.child, e.child $=$ null, $|e|=y$, where $0<y \leq \mid m$.child $\mid$, then the maximum length of the element in $B_{S}$ containing $e$ is $y$, thus, $\left|B_{S}\right|_{\max }=x+y$; If $\exists m=" \rightarrow "$, it means that $M\left[\right.$ m.child. $p_{i}>, M\left[\right.$ m.child. $p_{j}>, M\left[\right.$ m.child. $p_{i}>$

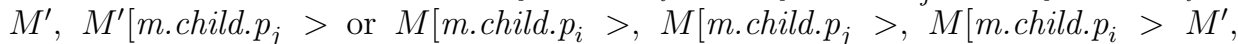
$\neg M^{\prime}\left[\right.$ m.child. $p_{j}>, i \neq j$ and $0<i, j \leq \mid$ m.child $\mid$, so the maximum length of the element in $B_{S}$ containing m.child is 1 , thus, $\left|B_{S}\right|_{\max }=x+1$.

By Theorem 3, in the process tree, the maximum length of the branch of the choice or concurrent structure is the sum of the number of leaf nodes of the child node of " $\rightarrow$ " and the number of nodes " $\times$ " and " $\wedge$ ".

Deviations are determined by judging whether the corresponding transitions of $\log$ activities are in a branch. For those log activities, we can obtain the branches that need to be concurrent with other branches. New activities that need to be inserted into the original models can also be obtained from the optimal alignment. The algorithm for determining the deviation position is given in the following.

Algorithm 3 calculates the deviation position for incomplete choice structures. We obtain the positions of $S F_{P}$ and $S L_{P}$ in the optimal alignment, and collect new activities. For activities between $S F_{P}$ and $S L_{P}$ in the optimal alignment, if the activity is a log activity and the corresponding transition is contained in the first choice activity set, we determine whether all transitions of the whole branch headed by this transition are log activities; if they are, we regard the first and last transitions of the branch as a deviation position.

$\gamma_{1}=\mid$\begin{tabular}{|l|l|l|l|l|l|}
$a$ & $t_{1}$ & $t_{5}$ & $t_{2}$ & $t_{4}$ & $b$ \\
\hline$a$ & $t_{1}$ & $>>$ & $t_{2}$ & $t_{4}$ & $b$
\end{tabular}

Figure 4. An optimal alignment $\gamma_{1}$ between $\sigma_{1}$ and $P N_{1}$

$\gamma_{2}=\mid$\begin{tabular}{|c|c|c|c|c|}
$a$ & $t_{6}$ & $t_{7}$ & $c$ & $b$ \\
\hline$a$ & $t_{6}$ & $t_{7}$ & $>>$ & $b$
\end{tabular}

Figure 5. An optimal alignment $\gamma_{2}$ between $\sigma_{2}$ and $P N_{1}$ 


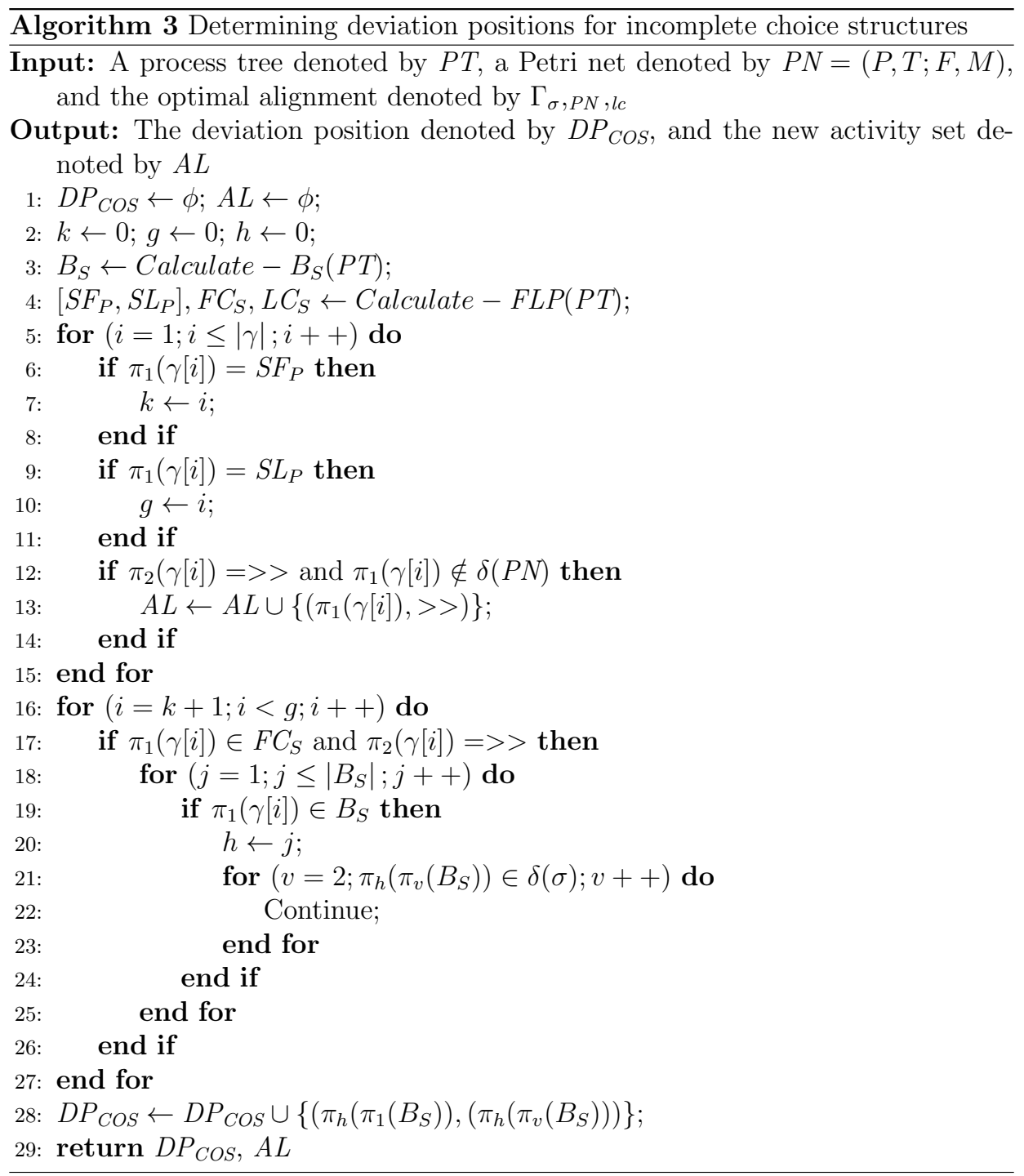

Figure 4 is an optimal alignment $\gamma_{1}$ between $\sigma_{1}$ and $P N_{1}$, and Figure 5 is an optimal alignment $\gamma_{2}$ between $\sigma_{2}$ and $P N_{1} . \sigma_{1}$ has transitions of choice structures with $t_{1}, t_{5}, t_{2}, t_{4}$, and the branch set of $P N_{1}$ is denoted as $B_{S}=\left\{\left\{t_{1}, t_{2}, t_{4}\right\},\left\{t_{1}, t_{3}, t_{4}\right\},\left\{t_{5}\right\}\right.$, $\left.\left\{t_{6}, t_{7}\right\}\right\}$. Its head-to-tail place is denoted as $\left[S F_{P}, S L_{P}\right]=\left[p_{2}, p_{6}\right]$. Its first choice activity set is denoted as $F C_{S}=\left\{t_{1}, t_{5}, t_{6}\right\}$, and the last choice activity set is denoted as $L C_{S}=\left\{t_{4}, t_{5}, t_{7}\right\}$. We can find that the branch $\left\{t_{5}\right\}$ occurs concurrently with the branch $\left\{t_{1}, t_{2}, t_{4}\right\}$ by traversing the optimal alignment. For $\gamma_{1},\left(t_{5},>>\right)$ is a $\log$ activity, and $t_{5}$ is both contained in first and last choice activity sets, so 
its deviation position denoted by $D P_{C O S}=\left\{\left(t_{5}, t_{5}\right)\right\}$. For $\gamma_{2}$, its new activity set denoted by $A L$ is $\{c\}$.

An algorithm is proposed to repair models with incomplete choice structures via logic Petri nets according to the deviation position in the following content.

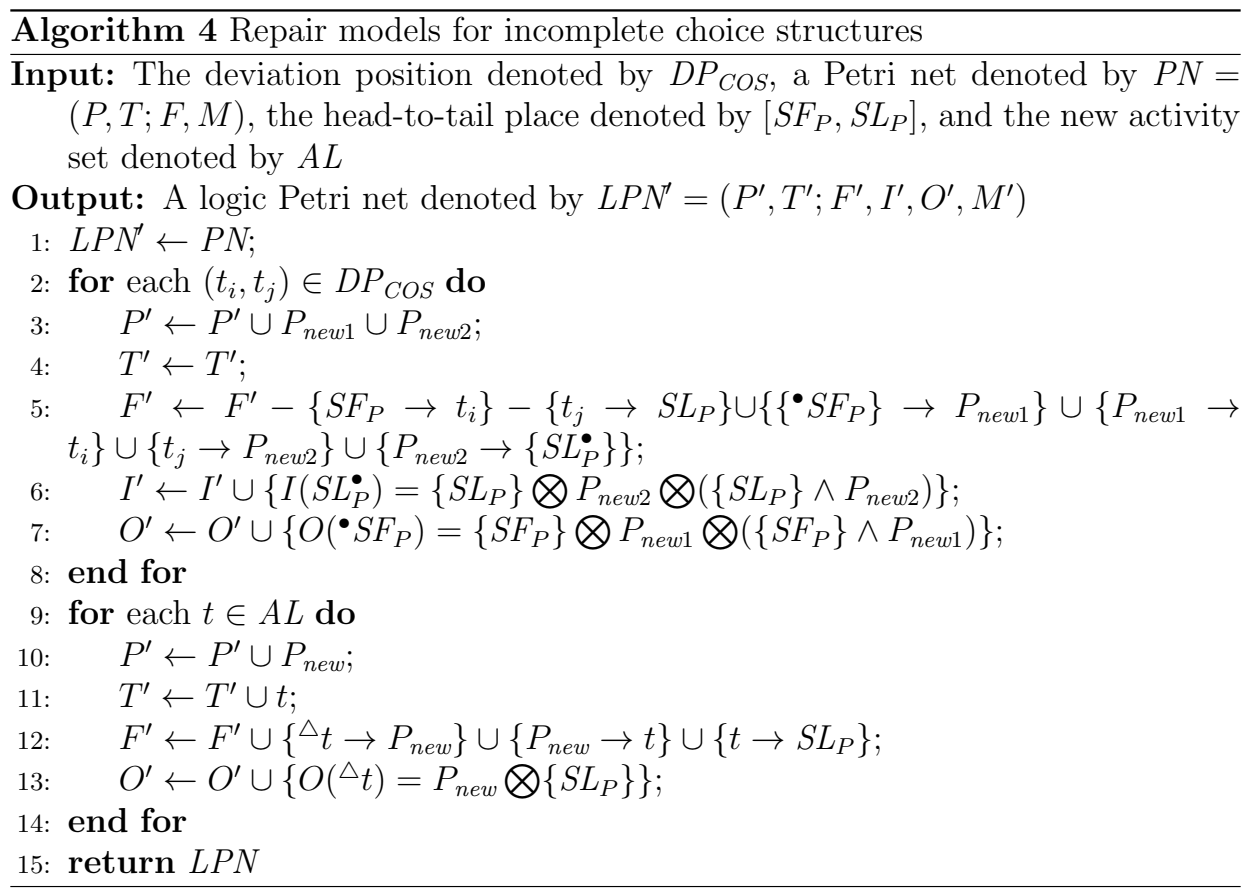

Algorithm 4 repairs models with incomplete choice structures. For each deviation position and new activity, we add different logic input and output transitions.

For $\sigma_{1}$ and $\sigma_{2}$, its deviation position and new activity set are denoted as $D P_{C O S}=$ $\left\{\left(t_{5}, t_{5}\right)\right\}$ and $A L=\{c\}$. For $D P_{C O S}=\left\{\left(t_{5}, t_{5}\right)\right\}$, we delete the arc from $p$ to $t_{5}$ and the arc from $t_{5}$ to $p^{\prime}$, where $p \in t^{\bullet} t_{5}$ and $p^{\prime} \in t_{5}^{\bullet}$. Then we add two places, and add the arc from $a$ to the new place and the arc from the new place to $t_{5}$, where $a \in^{\bullet} p$; we also add the arc from $t_{5}$ to the new place and the arc from the new place to $b$, where $b \in p^{\prime}$. We change $a$ to a logic output transition and change $b$ to a logic input transition. For $A L=\{c\},{ }^{\triangle} c=t_{7}$ and $c^{\triangle}=b$. We add a place and new transition $c$. Then we insert three arcs from $t_{7}$ to the new place and from the new place to $c$ and from $c$ to $b$ into the model. Besides, we change $t_{7}$ to a logic output transition. The repaired model by our approach denoted as $L P N_{2}$ is shown in Figure6. 


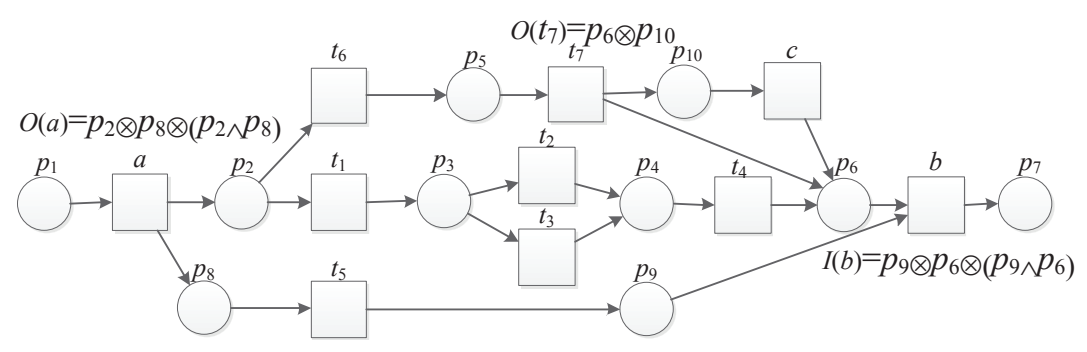

Figure 6 . The repaired model $L P N_{2}$ by our approach

\section{INCOMPLETE CONCURRENT STRUCTURES DEVIATION REPAIR}

This section presents a repair method of the model with incomplete concurrent structures. For incomplete concurrent structures, we propose concurrent relation sets, including head-to-tail transitions and concurrent activity sets. By comparing and analyzing the logic relations between activities in traces and transitions in concurrent activity sets, we can determine the deviation position of incomplete concurrent structures, and repair models with incomplete concurrent structures via logic Petri nets.

In the following, we use $P N=(P, T ; F, M)$ to denote a four-tuple Petri net, and use $P T$ to denote a process tree of $P N$.

Definition 15 (Head-to-tail transition). $\left[S F_{T}, S L_{T}\right]$ is called the head-to-tail transition of a concurrent structure, and $S F_{T}$ and $S L_{T}$ are called the head transition and the tail transition, respectively, where $S F_{T}=n . l s i b$ and $S L_{T}=n . r s i b, n=" \wedge$ " and $n \in P T$.

Theorem 4. For $n=$ " $\wedge$ ", if $\exists$ n.child $=m$ and $m$.child $=$ null, then $\{m\}$ is a branch.

Proof. If $\exists$ n. child $=m$ and $m$.child $=$ null, it means that the child node of $n$ is a leaf node, i.e., $\neg \exists$ m.child $\mid \rightarrow \bigoplus$, where $\bigoplus=\{\times, \rightarrow, \odot, \wedge\}$. Since $n=" \wedge "$, n.lsib $=$ $S F_{T}$ and $n . r s i b=S L_{T}$. We have $S F_{T}$. parent $=S L_{T}$.parent $=n$.parent $=$ " $\rightarrow$ ". Since $m=$ n.child and $m$.child $=$ null, it means that $S F_{T}, m$ and $S L_{T}$ fire in order, i.e., $S F_{T}=\bullet(\bullet m)$ and $S L_{T}=\left(m^{\bullet}\right)^{\bullet}$. Thus, $\{m\}$ is a branch.

If $\{m\}$ is a branch and $n=" \wedge "$, it means n.lsib $=S F_{T}$ and n.rsib $=S L_{T}$. We have $S F_{T}=\bullet(\bullet m)$ and $S L_{T}=\left(m^{\bullet}\right)^{\bullet}$. Thus, $\neg \exists$ m.child $\mid \rightarrow \bigoplus$ and " $\wedge " \mid \rightarrow m$, i.e., $m=$ n.child and $m$.child $=$ null.

By Theorem 4, for the node denoted by " $\wedge$ " of the process tree, if the child node of the node is a leaf node, then this child node is alone a branch in the concurrent structure.

Definition 16 (Concurrent activity set). First and last concurrent activity sets are called the concurrent activity set together. $F U_{S}$ and $L U_{S}$ denote the first and the 
last concurrent activity sets, respectively. If $n=" \wedge$ " and $n \in P T$, they need to satisfy:

1. if $n$.child $=m$, m.child $=$ null, then $F U_{S}=\{m\}$, and $L U_{S}=\{m\}$;

2. if $n|\rightarrow \ldots| \rightarrow m, m=$ " $\wedge$ " or $m=$ " $\times ", k=\mid$ m.child $\mid, q=$ n.child.lp and $m=q$. parent, then $F U_{S}=\left\{\right.$ m.child. $p_{1}$, m.child. $p_{2}, \ldots$, m.child. $\left.p_{k}\right\}$;

3. if $n|\rightarrow \ldots| \rightarrow m, m=$ "^" or $m=$ " $\times ", k=\mid$ m.child $\mid, q=$ n.child.rp and $m=q$.parent, then $L U_{S}=\left\{\right.$ m.child. $p_{1}$, m.child. $p_{2}, \ldots, m$. child. $\left.p_{k}\right\}$; and

4. if $n|\rightarrow \ldots| \rightarrow m, m=" \rightarrow$ ", and m.child.child $=$ null, then $F U_{S}=\{$ n.child.lp $\}$, and $L U_{S}=\{$ n.child.rp $\}$.

The algorithm of calculating head-to-tail transitions and concurrent activity sets is given in the following.

Algorithm 5 calculates the head-to-tail transition and the concurrent activity set. For each node $n$ of process tree, if $n$ is " $\wedge$ ", its head transition is the pre-set of the pre-set of the leftmost leaf node of $n$, and its tail place is the post-set of the post-set of the rightmost leaf node of $n$. For each child node of $n$, if its child node is a leaf node, then the child node of $n$ is contained in the first and last concurrent activity sets. If the child node $g$ of $n$ is " $\wedge$ " or " $\times$ ", and $g$ is the parent node of the leftmost or rightmost leaf node of $n$, then each child node of $g$ is contained in the first or last concurrent activity set. If $g$ is " $\rightarrow$ " and the child node of $g$ is a leaf node, then the leftmost leaf node of $g$ is contained in the first concurrent activity set, and the rightmost leaf node of $g$ is contained in the last concurrent activity set.

Figure 7 shows a Petri net model $P N_{2}$, and $P N_{2}$ contains concurrent structures. Let $\sigma_{3}=\left\langle a, t_{1}, t_{3}, t_{6}, e, c\right\rangle$ and $\sigma_{4}=\left\langle a, b, t_{4}, t_{5}, f, c\right\rangle$ be two traces. $P N_{2}$ has a concurrent structure with transitions $t_{1}, t_{2}, t_{3}, t_{4}, t_{5}, t_{6}$. In the trace $\sigma_{3}$, we have that $t_{1}, t_{3}$ and $t_{6}$ are concurrently enabled. For $P N_{2}$, according to Algorithm 1, we can calculate its branch set denoted by $B_{S}=\left\{\left\{t_{1}, t_{3}\right\},\left\{t_{2}, t_{3}\right\},\left\{t_{4}, t_{5}\right\},\left\{t_{6}\right\}\right\}$. It shows that the branches $\left\{t_{1}, t_{3}\right\}$ and $\left\{t_{6}\right\}$ can occur selectively with the branch $\left\{t_{4}, t_{5}\right\}$. In the original model $\mathrm{PN}_{2}$, all these branches need to occur. So this is an incomplete concurrent structure.

Figure 8 shows the process tree of $P N_{2}$ represented by $P T_{2}$. We have $P T_{2}=\rightarrow$ $\left(a, b, \wedge\left(\rightarrow\left(\times\left(t_{1}, t_{2}\right), t_{3}\right), \rightarrow\left(t_{4}, t_{5}\right), t_{6}\right), c\right)$. For $P T_{2}$, we can obtain the head-to-tail transition, the branch set and the concurrent activity set. Its head-to-tail transition is denoted as $\left[S F_{T}, S L_{T}\right]=[b, c]$, and its branch set is denoted as $B_{S}=$ $\left\{\left\{t_{1}, t_{3}\right\},\left\{t_{2}, t_{3}\right\},\left\{t_{4}, t_{5}\right\},\left\{t_{6}\right\}\right\}$. The first concurrent activity set is denoted as $F U_{S}=$ $\left\{t_{1}, t_{2}, t_{4}, t_{6}\right\}$, and the last concurrent activity set is denoted as $L U_{S}=\left\{t_{3}, t_{5}, t_{6}\right\}$.

For incomplete concurrent structures, we can obtain log activities and model activities from the optimal alignment. For those model activities, we can obtain branches that need to occur selectively with other branches. For log activities, we can obtain new activities that need to be inserted into the original model. The algorithm of determining deviation positions is given in the following content. 


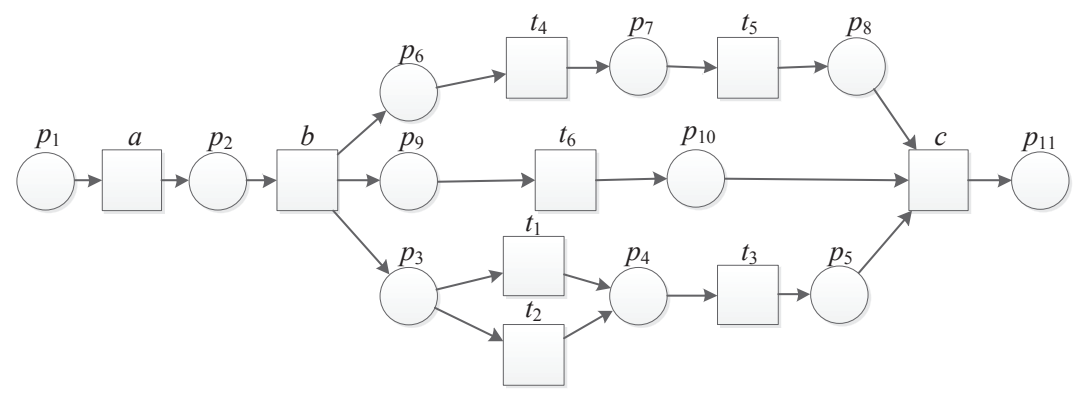

Figure 7. A Petri net model $P N_{2}$

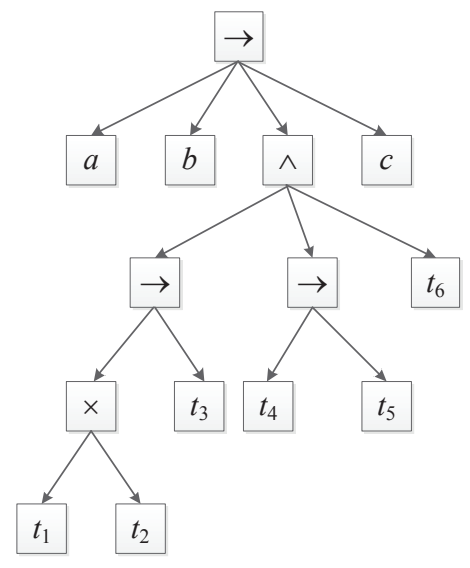

Figure 8. The process tree $P T_{2}$ of $P N_{2}$

Algorithm 6 calculates the deviation position for incomplete concurrent structures. We obtain the positions of $S F_{T}$ and $S L_{T}$ in the optimal alignment, and collect new activities. For activities between $S F_{T}$ and $S L_{T}$ in the optimal alignment, if the activity is a model activity and the corresponding transition is contained in the first concurrent activity set, we determine whether all transitions of the branch headed by this transition are model activities. If the corresponding activity of head transition is a model or synchronous activity, we regard the invisible or head transition and the first and last transitions in the branch as the deviation position.

An optimal alignment $\gamma_{3}$ between $\sigma_{3}$ and $P N_{2}$ is represented in Figure 9, and an optimal alignment $\gamma_{4}$ between $\sigma_{4}$ and $P N_{2}$ is shown in Figure 10. The branch set of $P N_{2}$ is $B_{S}=\left\{\left\{t_{1}, t_{3}\right\},\left\{t_{2}, t_{3}\right\},\left\{t_{4}, t_{5}\right\},\left\{t_{6}\right\}\right\}$. For $\gamma_{3},(>>, b),\left(>>, t_{4}\right)$ and $\left(>>, t_{5}\right)$ are model activities, and we can find that the branch $\left\{t_{4}, t_{5}\right\}$ can occur selectively with other branches by traversing the optimal alignment. For $\gamma_{4},\left(>>, t_{1}\right)$, 


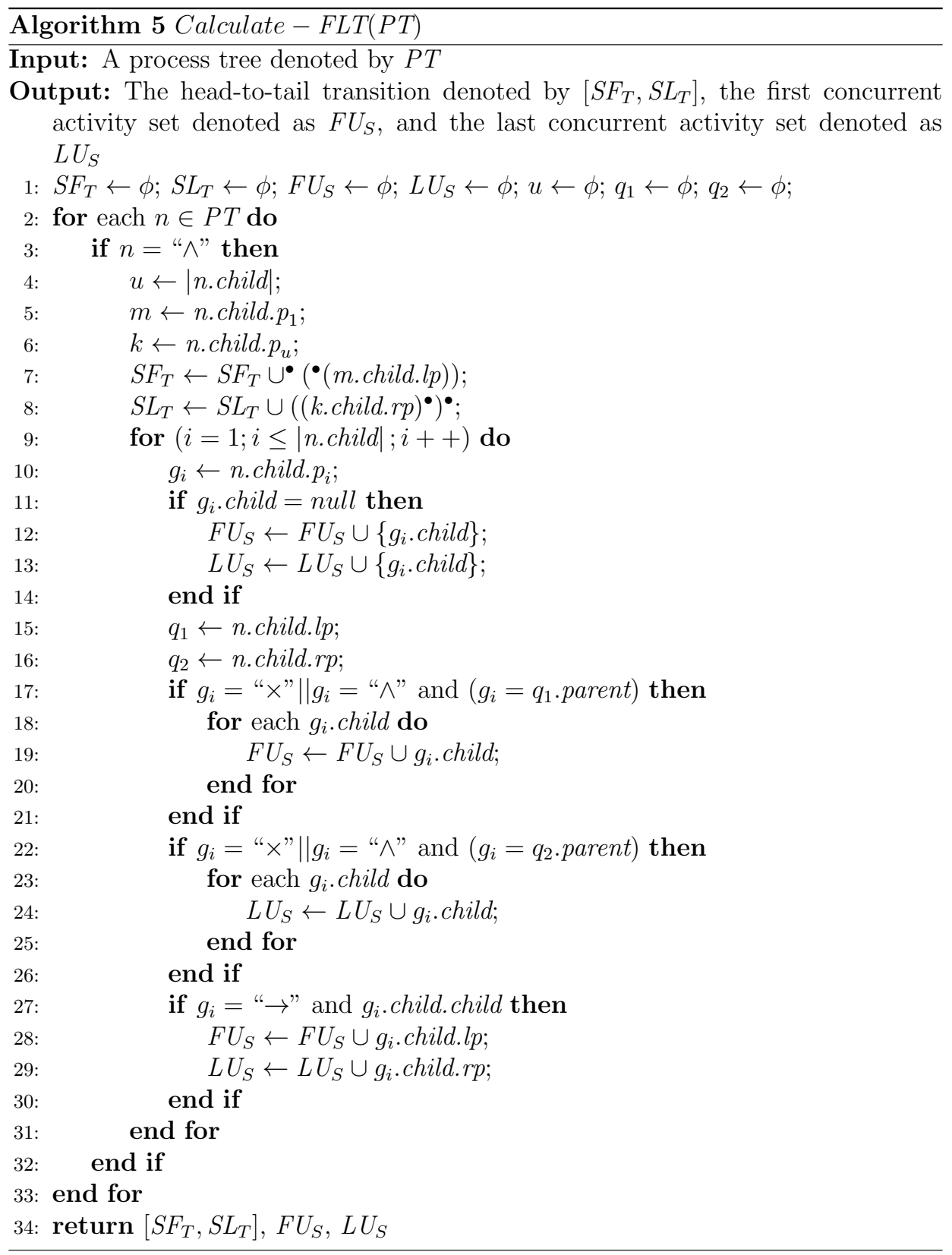




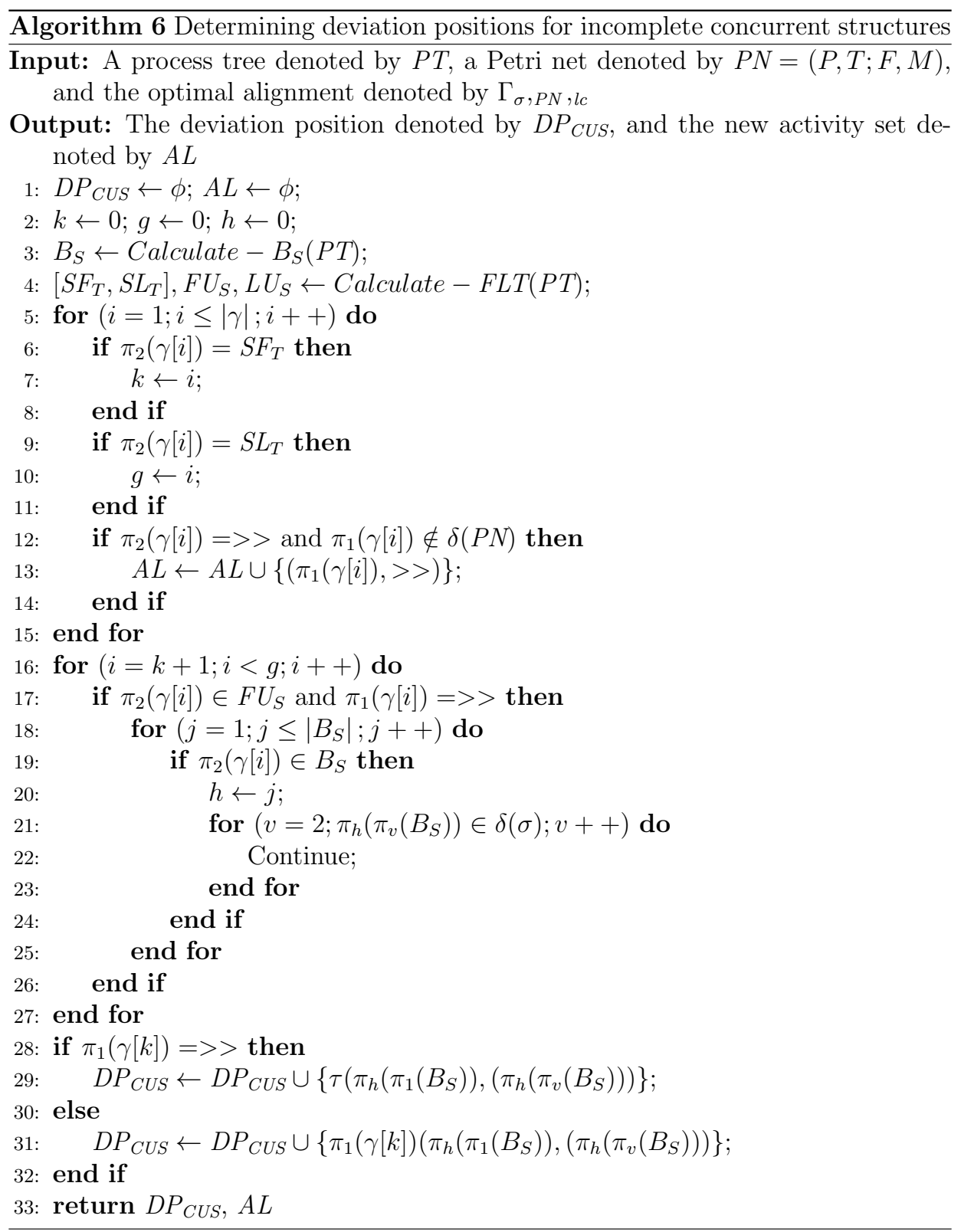


$\left(>>, t_{3}\right)$ and $\left(>>, t_{6}\right)$ are model activities, and we can find branches $\left\{t_{1}, t_{3}\right\}$ and $\left\{t_{6}\right\}$ occur selectively with other branches. Its head-to-tail transition is $\left[S F_{T}, S L_{T}\right]=$ $[b, c]$, its first concurrent transition is $F U_{S}=\left\{t_{1}, t_{2}, t_{4}, t_{6}\right\}$, and the last concurrent transition is $L U_{S}=\left\{t_{3}, t_{5}, t_{6}\right\}$. Because $\left\{t_{4}, t_{5}\right\}$ is a branch of $P N_{2}$, and the head transition $b$ also cannot fire, so $\left\{\tau\left(t_{4}, t_{5}\right)\right\}$ is a deviation. Because $\left\{t_{1}, t_{3}\right\}$ and $\left\{t_{6}\right\}$ are two branches of $P N_{2}$, and the head transition $b$ is a synchronous activity, so $\left\{b\left(t_{1}, t_{3}\right), b\left(t_{6}, t_{6}\right)\right\}$ is a deviation. So we obtain its deviation position is $D P_{C U S}=$ $\left.\left\{\tau\left(t_{4}, t_{5}\right)\right), b\left(t_{1}, t_{3}\right), b\left(t_{6}, t_{6}\right)\right\}$. Besides, $(e,>>)$ and $(f,>>)$ are log activities, the new transitions $e$ and $f$ need to be inserted into the model, and its new activity set is $A L=\{e, f\}$.

$\gamma_{3}=\left|\begin{array}{c|c|c|c|c|c|c|c|c|}a & >> & t_{1} & t_{3} & t_{6} & >> & >> & e & c \\ \hline a & b & t_{1} & t_{3} & t_{6} & t_{4} & t_{5} & >> & c\end{array}\right|$

Figure 9. An optimal alignment $\gamma_{3}$ between $\sigma_{3}$ and $P N_{2}$

$\gamma_{4}=\left|\begin{array}{|c|c|c|c|c|c|c|c|c|}a & b & t_{4} & t_{5} & >> & >> & >> & f & c \\ \hline a & b & t_{4} & t_{5} & t_{1} & t_{3} & t_{6} & >> & c\end{array}\right|$

Figure 10. An optimal alignment $\gamma_{4}$ between $\sigma_{4}$ and $P N_{2}$

Algorithm 7 repairs models for incomplete concurrent structures via logic Petri nets according to the deviation position. For different deviation positions, we add different logic input and output transitions.

For $\sigma_{3}, \sigma_{4}$ and $P N_{2}$, its deviation position is denoted by $D P_{C U S}=\left\{\tau\left(t_{4}, t_{5}\right)\right)$, $\left.b\left(t_{1}, t_{3}\right), b\left(t_{6}, t_{6}\right)\right\}$, and its new activity set is denoted by $A L=\{e, f\}$. For $\left.\tau\left(t_{4}, t_{5}\right)\right)$, we add an invisible transition to skip transition $b$, and add two arcs from the invisible transition to $p$ and $p^{\prime}$, where $p \in t_{1}$ and $p^{\prime} \in t_{6}$. For $b\left(t_{1}, t_{3}\right)$ and $b\left(t_{6}, t_{6}\right)$, we change the head transition $b$ to a logic output transition. For $A L=\{e, f\}$, we add two places and new transitions $e$ and $f$, and change the tail transition $c$ to a logic input transition. The model repaired by our approach denoted by $L P N_{3}$ is represented in Figure 11.

\section{EXPERIMENTAL EVALUATION}

This section will compare our repair approach with Fahland's approach, Knapsack's approach and Goldratt's approach. The data is from a routine examination and a CT index examination of a hospital in Qingdao, and event logs can be accessible at: https://pan.baidu.com/s/1Nx2vf82NYKB9TGbf8uYIFQ. The Fahland's approach is implemented in ProM6.6, which is a process mining tool with lots of plugins and can be available from: http://www.promtools.org/prom6/. The Goldratt's approach and Knapsack's approach are implemented in the DOS window and edited 


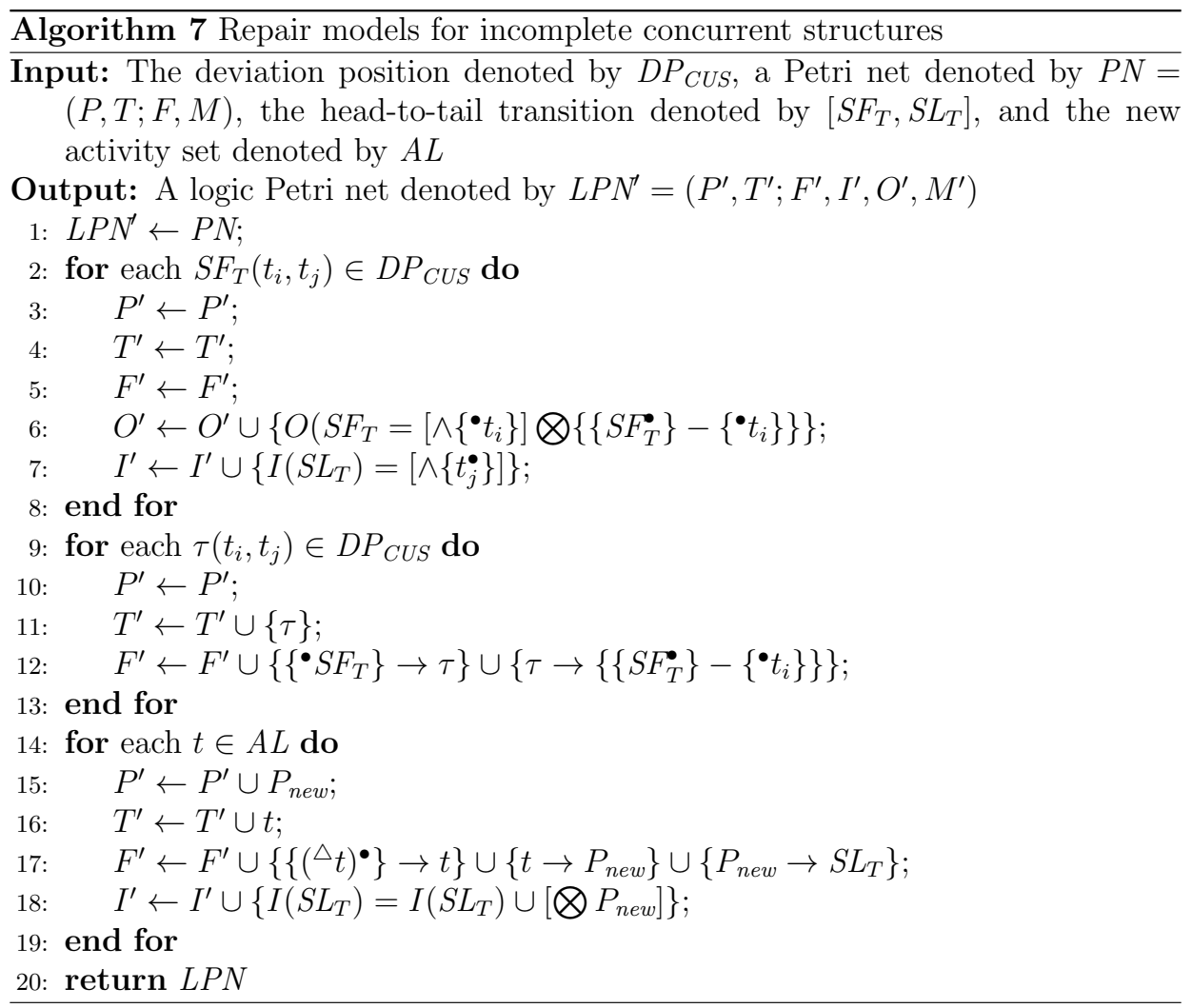

in ProM6.6. Since there are no corresponding experimental tools for mining and repairing logic Petri nets, the model repair and analysis of our repair approach use manual simulation in this paper.

\subsection{Experiment Data}

We take two business processes from a routine examination and a CT index examination in a hospital as examples. A hospital routine examination business process is represented in Figure 12. First, a patient makes an appointment in the hospital and pays for an appointment. Then he (or she) can get a number and wait for his (or her) order. After that, a doctor will check what the patient needs by the routine examination. There are five types of examinations and the patient can do one of them, i.e., the electrocardiogram, the abdominal ultrasound, the lung function examination, the blood glucose and lipid, and the liver and kidney examination. Finally, a doctor will diagnose and cure disease according to examinations. Figure 13 shows a hospital CT index examination business process. First, a patient goes to the information desk to consult some related problems and makes an appointment. 


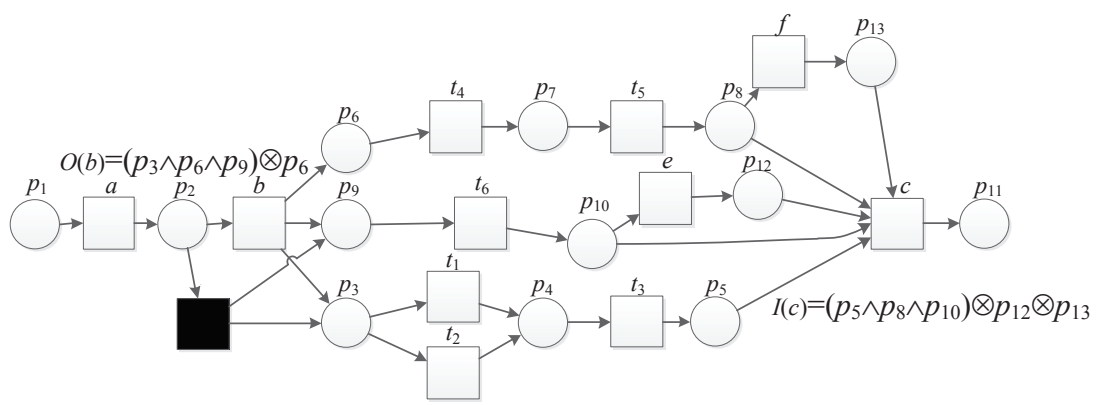

Figure 11. The repaired model $L P N_{3}$ by our approach

Then he (or she) will have an outpatient examination and take a brain CT and a head CT. Besides, a doctor checks for four symptoms, i.e., the sinusitis, the brain damage, the cerebral infarction, and the intracranial tumor. After that, the patient needs a surgery and is hospitalized for tests.

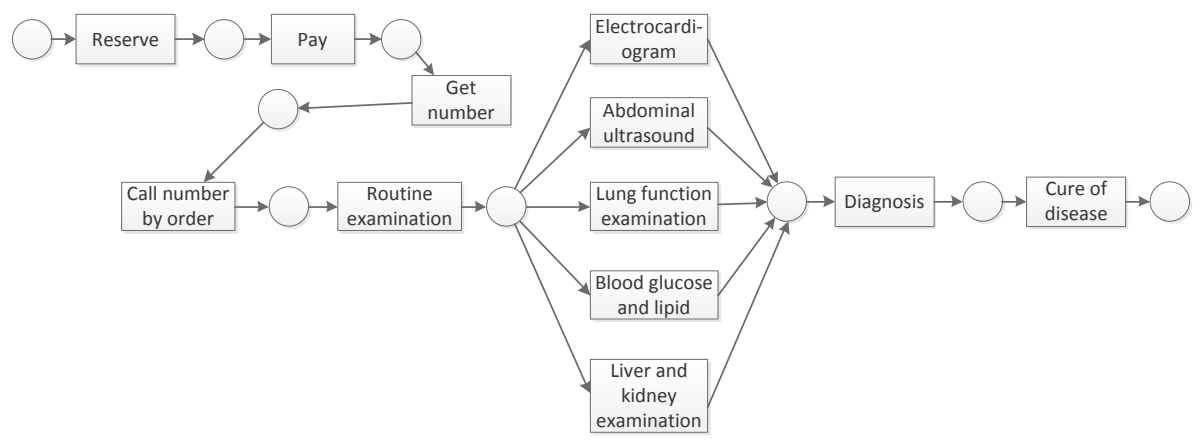

Figure 12. A hospital routine examination business process

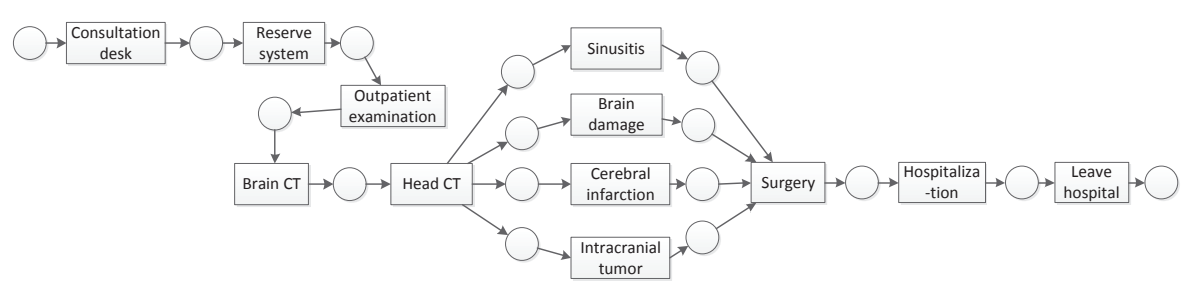

Figure 13. A hospital CT index examination business process

However, some event logs deviating from the original model are generated in the real process model systems. For example, in the hospital routine examination 
business process, a patient can do four tests at once, i.e., the liver and kidney examination, the lung function examination, the blood glucose and lipid, and the electrocardiogram; the patient also can carry out the abdominal ultrasound and the gynecological examination together. In the hospital CT index examination business process, the patient may only have a brain examination, he (or she) is tested for only three conditions, i.e., the intracranial tumor, the brain damage, and the cerebral infarction; the patient also can take medicines after the sinusitis examination. In those situations, the process models need to be repaired, the choice structure needs to be repaired to an incomplete choice structure, and the concurrent structure needs to be repaired to an incomplete concurrent structure. These two repaired structures can describe both choice and concurrent structures. Petri net-based models cannot simply and accurately express those structures, and we can repair model based on logic Petri nets.

\subsection{Model Repair}

For event logs of a hospital routine examination business process, we first filter out event logs that are significantly deviated from the examination business process. And according to the preprocessed ten sets of event logs (as shown in Table 1), the process model (as shown in Figure 12) is repaired based on four model repair methods. Table 1 records the specific information of activities in event logs and the number of deviations.

\begin{tabular}{|l|r|r|r|r|r|}
\hline Logs & Traces & Events & Transitions & Length & Deviations \\
\hline$L_{1}$ & 100 & 1020 & 13 & $8-11$ & 220 \\
\hline$L_{2}$ & 200 & 2103 & 13 & $8-12$ & 503 \\
\hline$L_{3}$ & 300 & 3143 & 13 & $8-12$ & 743 \\
\hline$L_{4}$ & 400 & 4306 & 13 & $8-12$ & 1106 \\
\hline$L_{5}$ & 500 & 5447 & 13 & $8-12$ & 1447 \\
\hline$L_{6}$ & 600 & 6129 & 13 & $8-11$ & 1378 \\
\hline$L_{7}$ & 700 & 7169 & 13 & $8-11$ & 1570 \\
\hline$L_{8}$ & 800 & 8179 & 13 & $8-11$ & 1780 \\
\hline$L_{9}$ & 900 & 9169 & 13 & $8-11$ & 1970 \\
\hline$L_{10}$ & 1000 & 10167 & 13 & $8-11$ & 2168 \\
\hline
\end{tabular}

Table 1. Event $\operatorname{logs} L_{1}-L_{10}$ of a routine examination business process

Our proposed approach is compared with Fahland's approach, Knapsack's approach and Goldratt's approach to illustrate its correctness and effectiveness. For incomplete choice structures, the Fahland's approach repairs the choice structure by adding loop structures, and adds invisible transitions to skip transitions that are not enabled. The Goldratt's method and Knapsack's method add different self-loops of repeat transitions to improve the fitness of process models. For incomplete concurrent structures, the Fahland's approach collects new transitions as a sub-log and inserts it into the original model, and skips transitions that are not 
enabled by adding invisible transitions. Besides, the Goldratt's method and Knapsack's method also add many invisible transitions to make repaired models better replay event logs generated in real systems. These invisible transitions and repeat transitions increase the uncertainty of the operation of the model, which leads to a poor performance of the model. These models cannot represent the logic relation among activities in incomplete choice structures and incomplete concurrent structures well, and most of them deviate from original structures of process models. It does not take advantage of the application and extension of process models.

For Figure 12 and Table 1, models repaired by four approaches are represented in Figures 14, 15, 16 and 17, respectively. The model repaired by Fahland's method adds a loop structure to make transitions in the choice structure fire simultaneously. The models repaired by Goldratt's approach and Knapsack's approach add different self-loops of single transitions. Our approach does not add any repeat transitions and does not add loop structures to change the basic structure of the original model. The model repaired by our method contains 3 logic transitions, the logic input function is $I$ (Diagnosis $)=p_{10} \otimes p_{11} \otimes p_{13} \otimes p_{14} \otimes\left(p_{10} \wedge p_{11} \wedge p_{13} \wedge p_{14}\right)$, and the logic output functions are $O($ Routine examination $)=p_{6} \otimes p_{7} \otimes p_{8} \otimes p_{9} \otimes\left(p_{6} \wedge p_{7} \wedge p_{8} \wedge p_{9}\right)$ and $O($ Abdominal ultrasound $)=p_{12} \otimes p_{13}$. Our repaired model can describe the logic relation among transitions of incomplete choice structures. These input and output functions limit the enablement of transitions in the model, so the model generates fewer traces that are not included in event logs.

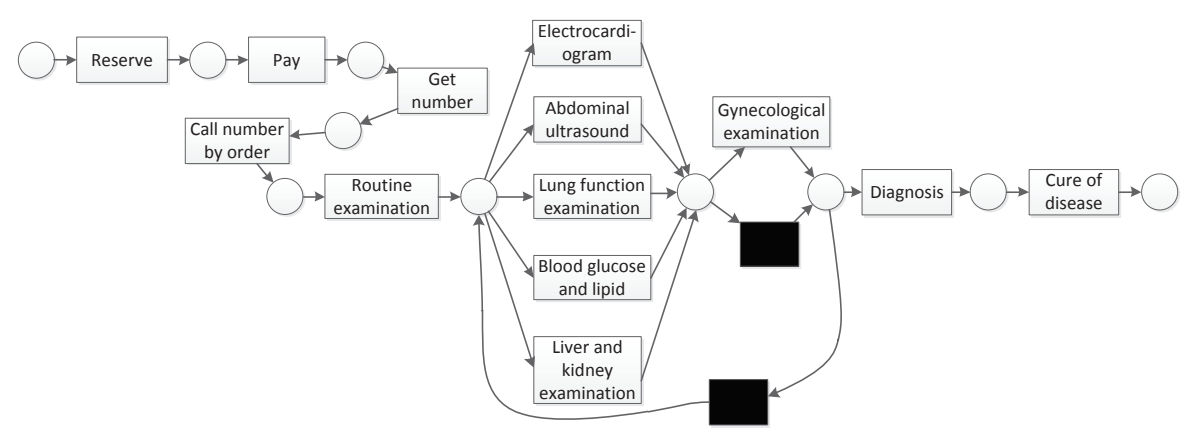

Figure 14. The repaired routine examination model by Fahland's method

Compared with the original model, the addition results of four repair models are represented in Table 2. As shown in Table 2, Goldratt's method adds the largest number of transitions (including invisible transitions), and our method only adds one transition without invisible transitions. The number of added repeat transitions by Goldratt's method and Knapsack's method are 6 and 4, respectively. Compared with other three repair methods, our method adds the least transitions, and does not add invisible transitions and repeat transitions. 


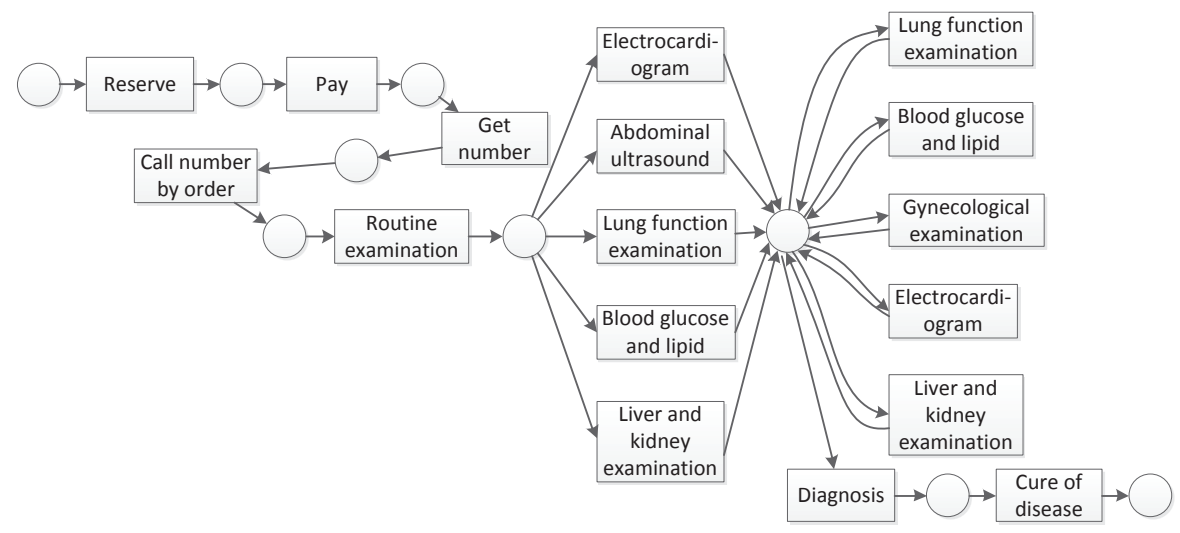

Figure 15. The repaired routine examination model by Knapsack's method

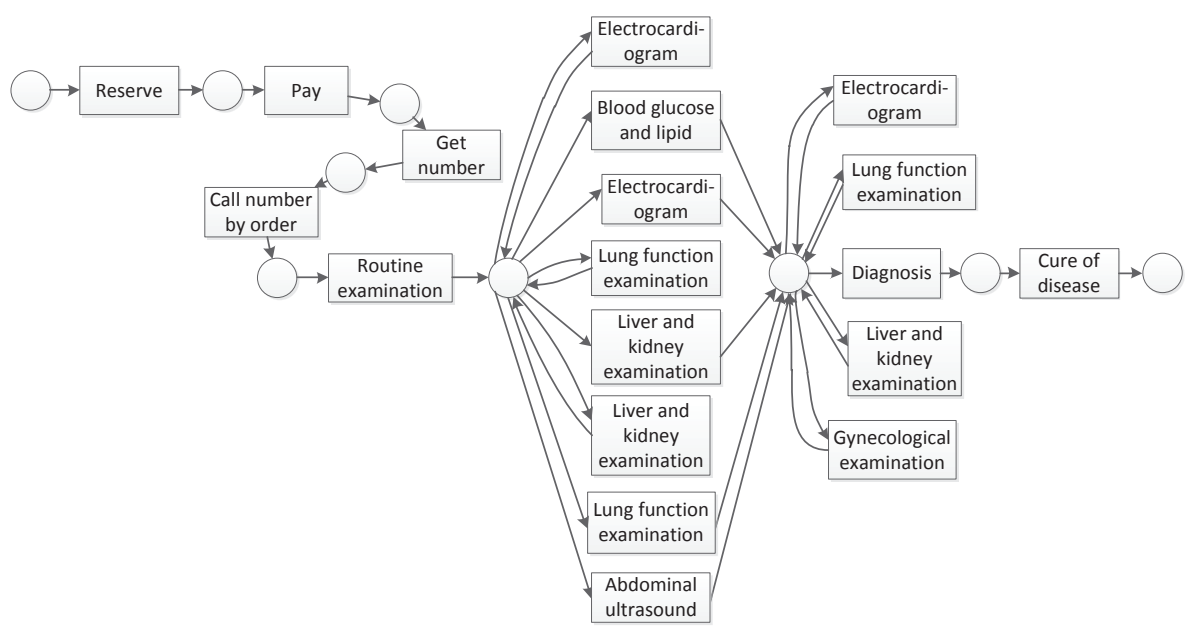

Figure 16. The repaired routine examination model by Goldratt's method

\begin{tabular}{|l|r|r|r|r|}
\hline Four Repair Methods & Added $|P|$ & Added $|T+\tau|$ & Added $|F|$ & Added Repeat $|T|$ \\
\hline Our method & 7 & 1 & 9 & 0 \\
\hline Fahland's method & 1 & 3 & 6 & 0 \\
\hline Goldratt's method & 0 & 7 & 14 & 6 \\
\hline Knapsack's method & 0 & 5 & 10 & 4 \\
\hline
\end{tabular}

Table 2. The addition results of Figures 14, 15, 16 and 17 


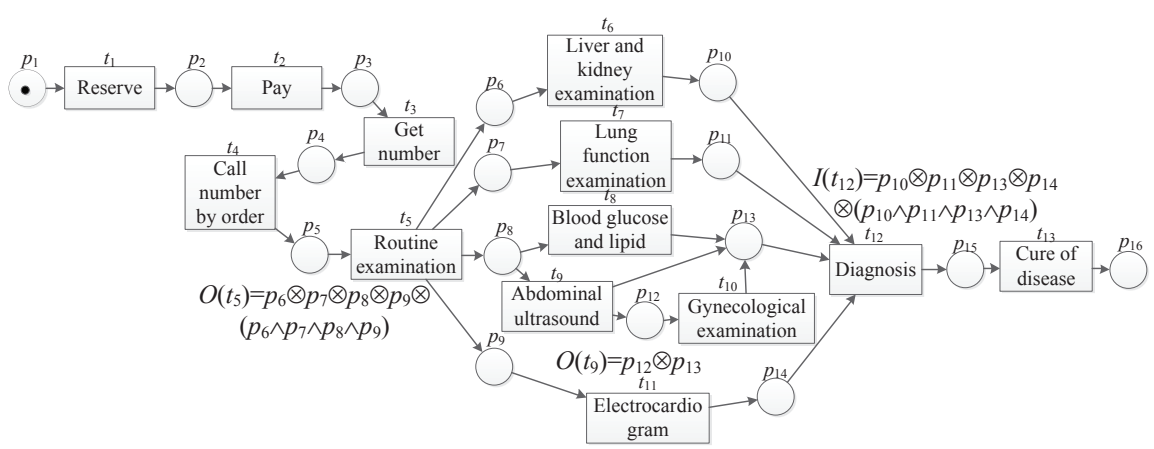

Figure 17. The repaired routine examination model by our approach

We process event logs of a CT index examination business process according to the same data filtering method. The filtered ten sets of event logs are described in Table 3. The process model (as shown in Figure 13) is repaired based on ten sets of event logs. The information of activities in event logs and the number of deviations are recorded in Table 3 .

\begin{tabular}{|l|r|r|r|c|r|}
\hline Logs & Traces & Events & Transitions & Length & Deviations \\
\hline$L_{11}$ & 100 & 1166 & 13 & $10-12$ & 38 \\
\hline$L_{12}$ & 200 & 2366 & 13 & $9-12$ & 38 \\
\hline$L_{13}$ & 300 & 3366 & 13 & $9-12$ & 238 \\
\hline$L_{14}$ & 400 & 4566 & 13 & $9-12$ & 238 \\
\hline$L_{15}$ & 500 & 5765 & 13 & $9-12$ & 238 \\
\hline$L_{16}$ & 600 & 6965 & 13 & $9-12$ & 238 \\
\hline$L_{17}$ & 700 & 8165 & 13 & $9-12$ & 238 \\
\hline$L_{18}$ & 800 & 9100 & 13 & $9-12$ & 573 \\
\hline$L_{19}$ & 900 & 10300 & 13 & $9-12$ & 573 \\
\hline$L_{20}$ & 1000 & 11500 & 13 & $9-12$ & 573 \\
\hline
\end{tabular}

Table 3 . Event $\operatorname{logs} L_{11}-L_{20}$ of a CT index examination business process

Four repaired models repaired by three classic methods and our repair method are shown in Figure 18, 19, 20 and 21, respectively. Fahland's method inserts many invisible transitions into the model to skip concurrent transitions that cannot fire. Although it makes activities in event logs be well replayed in the model, it also reduces the precision and simplicity of the model. Goldratt's approach inserts activities deviating from the model into the original model by means of self-loops. Knapsack's approach repairs the model in the same way as Goldratt's approach with different constraints. Besides, these two methods add a lot of invisible transitions. The model repaired by our approach reduces the degree of uncertainty of transition firing and has a high simplicity of net structures. Besides, it contains one logic input function and one logic output function, and they are 
$I($ Surgery $)=\left(p_{10} \wedge p_{11} \wedge p_{12} \wedge p_{13}\right) \bigotimes p_{14} \otimes\left(p_{10} \wedge p_{11} \wedge p_{12}\right)$ and $O($ Head $C T)=$ $\left(p_{6} \wedge p_{7} \wedge p_{8} \wedge p_{9}\right) \bigotimes p_{9}$. The repaired model by our approach can describe the logic relation among transitions of incomplete concurrent structures, and it does not generate additional transitions to increase the uncertainty of the operation of the model.

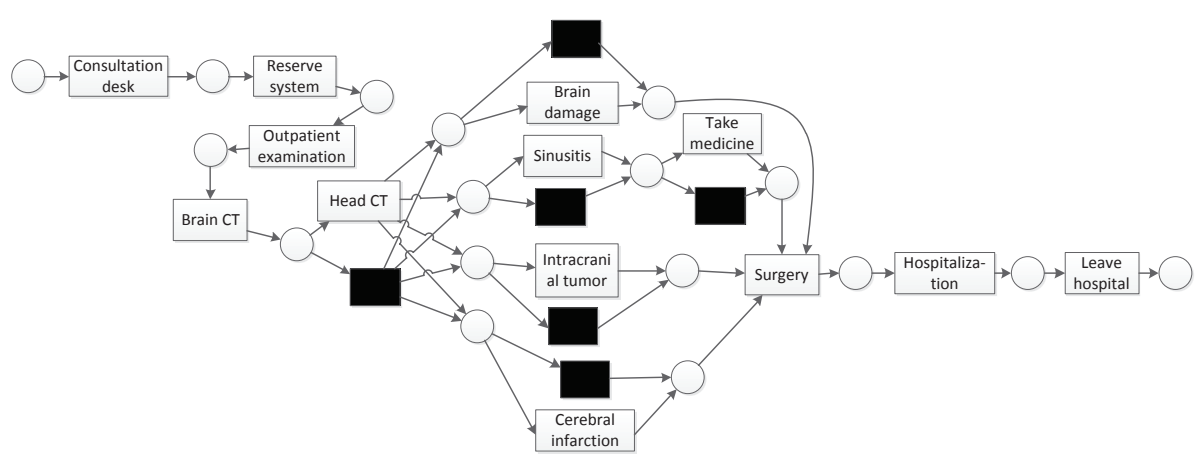

Figure 18. The repaired CT index examination model by Fahland's method

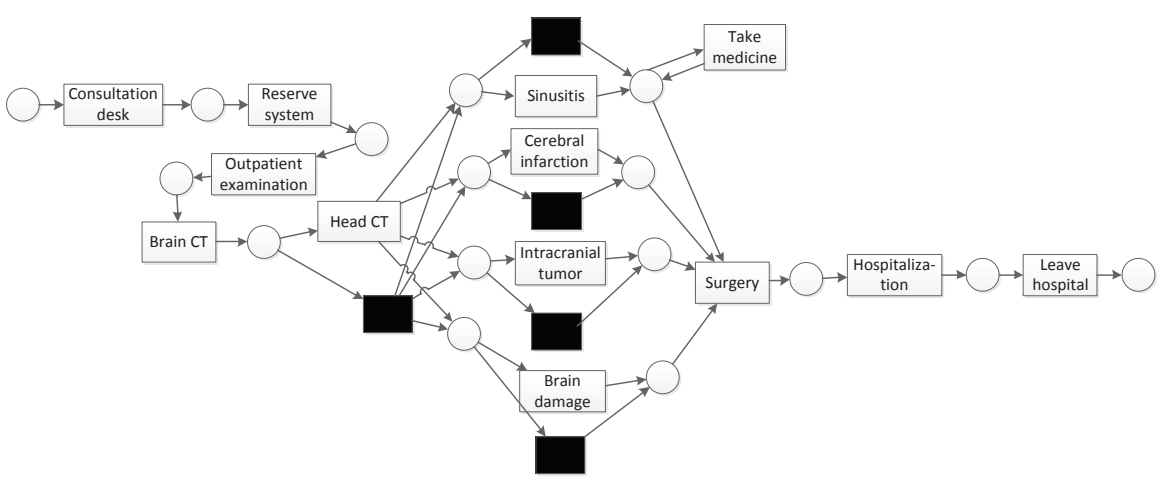

Figure 19. The repaired CT index examination model by Knapsack's method

Table 4 records addition results of four repair methods. Fahland's method adds the maximum number of transitions, including invisible transitions. The addition result of our repaired model is best, and the other three repaired models add a lot of invisible transitions and repeat transitions.

\subsection{Performance Analysis}

The sub-section describes the performance analysis of four repaired models combining with event $\operatorname{logs} L_{1}-L_{20}$. Fitness is the proportion of traces that the model 


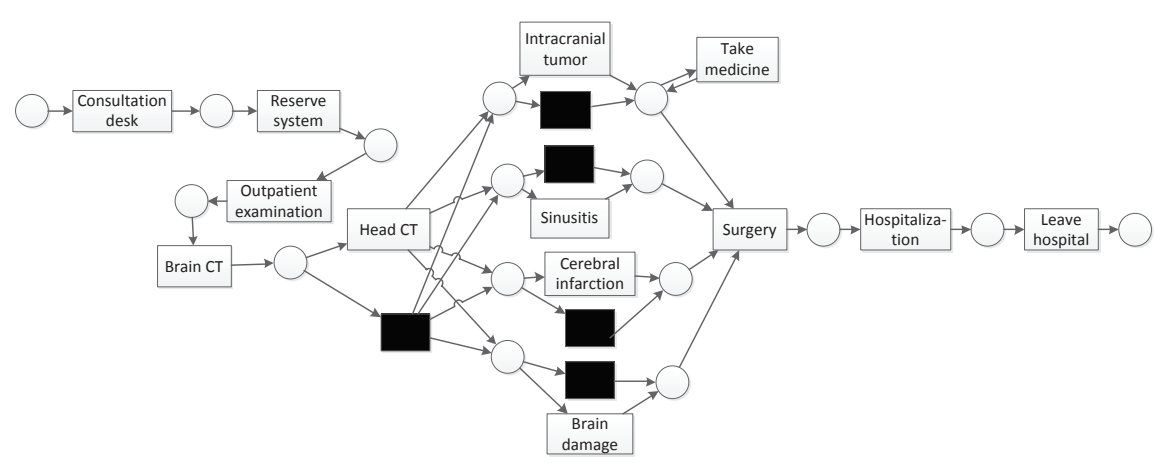

Figure 20. The repaired CT index examination model by Goldratt's method

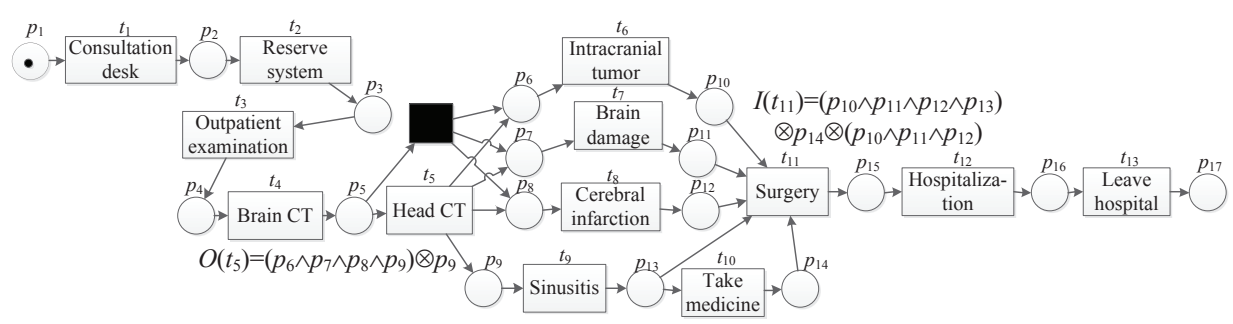

Figure 21. The repaired CT index examination model by our approach

can completely replay in the event log. A model with high fitness allows behaviors described in the event log to occur. If a model is precise, it does not allow too many activities that are not described in event logs to appear in the model. The formula for calculating fitness and precision of logic Petri nets are proposed in [19]. We consider the structure of the model and the repeatability of activities, and calculate the simplicity of net-based structures according to the method proposed in [18].

For four repaired models, we obtain the degree of fitness between models and event $\operatorname{logs} L_{1}-L_{20}$ represented in Table 5 and Table 6. As shown in Table 5 , for our approach, Fahland's approach and Goldratt's approach, the fitness be-

\begin{tabular}{|l|r|r|r|r|}
\hline Four Repair Methods & Added $|P|$ & Added $|T+\tau|$ & Added $|F|$ & Added Repeat $|T|$ \\
\hline Our method & 1 & 2 & 7 & 0 \\
\hline Fahland's method & 1 & 7 & 17 & 0 \\
\hline Goldratt's method & 0 & 6 & 15 & 1 \\
\hline Knapsack's method & 0 & 6 & 15 & 1 \\
\hline
\end{tabular}

Table 4. The addition results of Figures 18, 19, 20, and 21 
tween these repaired models and event $\operatorname{logs} L_{1}-L_{10}$ are all 1 . However, the fitness value of the model repaired by Goldratt's method is slightly lower than that of the other methods. As shown in Table 6, the degree of fitness between these four repaired models and event $\operatorname{logs} L_{11}-L_{20}$ are all 1 . In general, the fitness of these four methods is very high, and those four repaired models all have a high fitness.

\begin{tabular}{|l|c|c|c|c|c|c|c|c|c|c|}
\hline Four Repair Methods & $L_{1}$ & $L_{2}$ & $L_{3}$ & $L_{4}$ & $L_{5}$ & $L_{6}$ & $L_{7}$ & $L_{8}$ & $L_{9}$ & $L_{10}$ \\
\hline Fahland's method & 1 & 1 & 1 & 1 & 1 & 1 & 1 & 1 & 1 & 1 \\
\hline Our method & 1 & 1 & 1 & 1 & 1 & 1 & 1 & 1 & 1 & 1 \\
\hline Goldratt's method & 1 & 1 & 1 & 1 & 1 & 1 & 1 & 1 & 1 & 1 \\
\hline Knapsack's method & 1 & 0.9884 & 0.9884 & 0.9723 & 0.9710 & 1 & 1 & 1 & 1 & 1 \\
\hline
\end{tabular}

Table 5 . The fitness between different models and event $\operatorname{logs} L_{1}-L_{10}$

\begin{tabular}{|l|c|c|c|c|c|c|c|c|c|c|}
\hline Four Repair Methods & $L_{1}$ & $L_{2}$ & $L_{3}$ & $L_{4}$ & $L_{5}$ & $L_{6}$ & $L_{7}$ & $L_{8}$ & $L_{9}$ & $L_{10}$ \\
\hline Fahland's method & 1 & 1 & 1 & 1 & 1 & 1 & 1 & 1 & 1 & 1 \\
\hline Our method & 1 & 1 & 1 & 1 & 1 & 1 & 1 & 1 & 1 & 1 \\
\hline Goldratt's method & 1 & 1 & 1 & 1 & 1 & 1 & 1 & 1 & 1 & 1 \\
\hline Knapsack's method & 1 & 1 & 1 & 1 & 1 & 1 & 1 & 1 & 1 & 1 \\
\hline
\end{tabular}

Table 6 . The fitness between different models and event $\operatorname{logs} L_{11}-L_{20}$

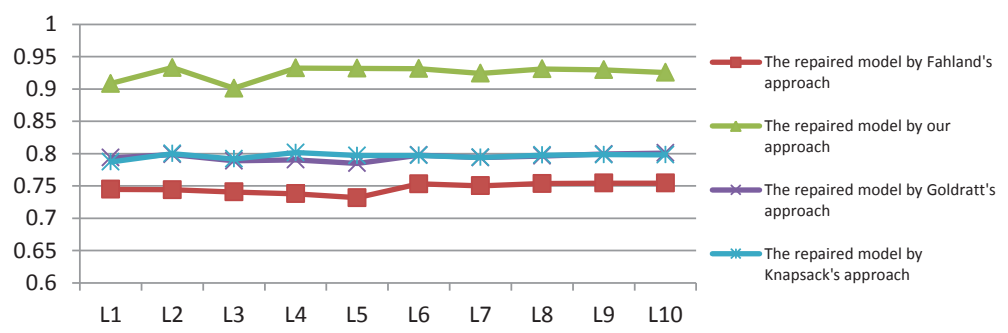

Figure 22. The precision between different models and event logs $L_{1}-L_{10}$

The results of precision between models proposed by four repair methods and event $\operatorname{logs} L_{1}-L_{20}$ are represented in Figure 22 and Figure 23. As shown in Figure 22, the model repaired by our approach has the highest precision, significantly higher than Knapsack's approach, Goldratt's approach and Fahland's approach. As shown in Figure 23, the model repaired by our approach also has the highest precision, and it has a clear performance advantage than the other three repair methods. In general, the precision of our repaired model for incomplete choice structures or incomplete concurrent structures is significantly higher than that of the three other classic repair methods. Our proposed method greatly improves the 


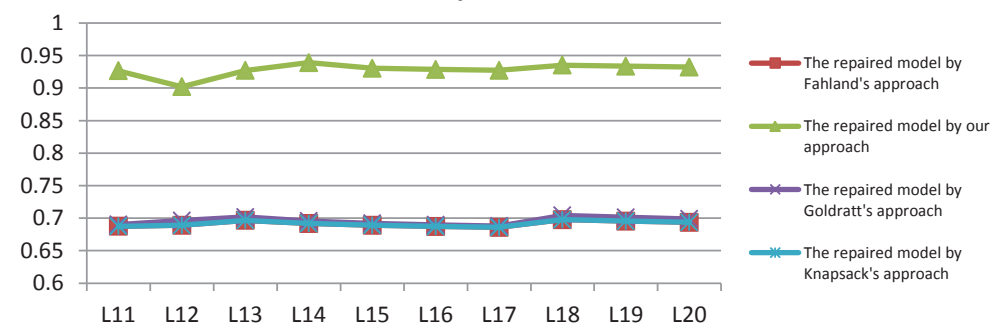

Figure 23. The precision between different models and event logs $L_{11}-L_{20}$

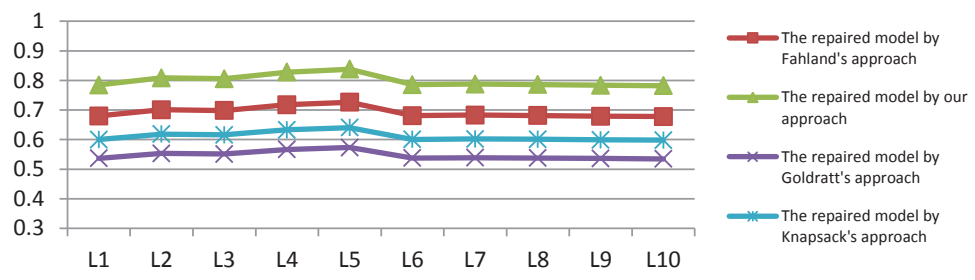

Figure 24. The simplicity between different models and event $\operatorname{logs} L_{1}-L_{10}$

precision of the process models and also improves the performance of the models.

The simplicity of net structures is obtained by calculating the sum of the proportion of events of per trace in the total number of transitions of models. The results of simplicity of four repair approach combining with event $\operatorname{logs} L_{1}-L_{20}$ are represented in Figure 24 and Figure 25. As shown in Figure 24, the simplicity of the repaired model by our approach is highest, followed by Fahland's approach. These two methods are higher than Knapsack's approach and Goldratt's approach. As shown in Figure 25, our repair approach has the highest simplicity of net structures, higher than Goldratt's approach and Knapsack's approach. By comparison, the repaired model by Fahland's approach has the lowest simplicity of net structures. In terms of net structures, our repair approach has the highest simplicity of net structures, higher than Goldratt's approach, Knapsack's approach

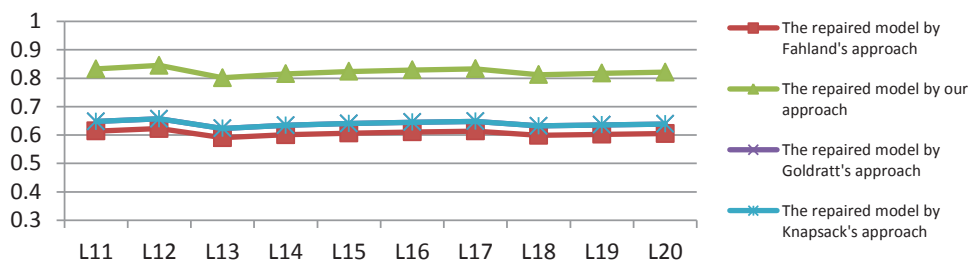

Figure 25. The simplicity between different models and event $\operatorname{logs} L_{11}-L_{20}$ 
and Fahland's approach. Therefore, for business processes with incomplete concurrent and choice structures, our method not only enables the model to have a high precision and fitness, but also improves the simplicity of net structures of the model.

\section{CONCLUSIONS}

In the paper, the repair method for process models with incomplete choice and concurrent structures is proposed via logic Petri nets. Current model repair methods tend to change the choice structure to a loop structure, and generate a large number of invisible and repeat transitions, or add self-loops of transitions to improve fitness. The concepts of choice relation sets and concurrent relation sets are presented based on process trees. For incomplete choice structures, we judge whether the corresponding transitions of log activities are contained in a whole branch, and determine deviation positions based on choice activity sets. For incomplete concurrent structures, we find deviations by judging whether the corresponding transitions of model activities are contained in a whole branch and whether the head-to-tail transition is a synchronous activity. Then we determine deviation positions based on concurrent activity sets, and repair models via logic Petri nets according to different deviation positions. Through the simulation experiment, we prove that our repair approach greatly improves the precision and simplicity of the model, and the fitness is still very high. It also can describe the logic relation among transitions in incomplete choice and concurrent structures correctly. However, process trees can only represent Petri nets with block-distributed structures. For those process models that cannot be represented by process trees, we will further study how to determine the deviation position through other algorithms.

\section{Acknowledgements}

This work was supported in part by the National Natural Science Foundation of China under Grant 61903229 and Grant 61973180, in part by the Key Research and Development Program of Shandong Province under Grant 2018GGX101011, and in part by the Natural Science Foundation of Shandong Province under Grant ZR2018MF001 and Grant ZR2019BF004.

\section{REFERENCES}

[1] QI, H. D.-Du, Y. Y.—Liu, W.: Process Model Repairing Method Based on Reachable Markings. Journal of Shandong University of Science and Technology (Natural Science), Vol. 36, 2017, No. 1, pp. 118-124. 
[2] Conforti, R.-Dumas, M.-García-Bañuelos, L.-La Rosa, M.: BPMN Miner: Automated discovery of BPMN Process Models with Hierarchical Structure. Information Systems, Vol. 56, 2016, pp. 284-303, doi: 10.1016/j.is.2015.07.004.

[3] Van der Aalst, W. M. P.- - ter Hofstede, A. H. M.: YAWL: Yet Another Workflow Language. Information Systems, Vol. 30, 2004, No. 4, pp. 245-275, doi: 10.1016/j.is.2004.02.002.

[4] Liu, G. J.: Complexity of the Deadlock Problem for Petri Nets Modeling Resource Allocation Systems. Information Sciences: An International Journal, Vol. 363, 2016, Iss. C, pp. 190-197, doi: 10.1016/j.ins.2015.11.025

[5] Wang, Y. Y.-Du, Y. Y.: Conformance Checking Based on Extended Footprint Matrix. Journal of Shandong University of Science and Technology (Natural Science), Vol. 37, 2018, No. 2, pp. 9-15.

[6] Van der Aalst, W. M. P.-Weijters, A. J. M. M.-Maruster, L.: Workflow Mining: Discovering Process Model from Event Logs. IEEE Transactions on Knowledge and Data Engineering, Vol. 16, 2004, No. 9, pp. 1128-1142, doi: 10.1109/TKDE.2004.47.

[7] Weijters, A. J. M. M.—VAn Der Aalst, W. M. P.: Rediscovering Workflow Models from Event-Based Data Using Little Thumb. Integrated Computer-Aided Engineering, Vol. 10, 2003, No. 2, pp. 151-162.

[8] Adriansyah, A.-Munoz-Gama, J.-Carmona, J.-Van Dongen, B. F.van Der Aalst, W. M. P.: Aligning Based Precision Checking. In: La Rosa, M., Soffer, P. (Eds.): Business Process Management Workshops (BPM 2012). Springer, Berlin, Heidelberg, Lecture Notes in Business Information Processing, Vol. 132, pp. 137-149, doi: 10.1007/978-3-642-36285-9_15.

[9] Leemans, S. J. J.-Fahland, D.—van der Aalst, W. M. P.: Scalable Process Discovery and Conformance Checking. Software and Systems Modeling, Vol. 17, 2018, pp. 599-631, doi: 10.1007/s10270-016-0545-x.

[10] Alizadeh, M.-Lu, X.-Fahland, D.-Yanonne, N.VAN DER AAlst, W. M. P.: Linking Data and Process Perspectives for Conformance Analysis. Computers and Security, Vol. 73, 2018, pp. 172-193, doi: 10.1016/j.cose.2017.10.010.

[11] Rozinat, A.- - van Der Aalst, W. M. P.: Conformance Checking of Processes Based on Monitoring Real Behavior. Information Systems, Vol. 33, 2008, No. 1, pp. 64-95, doi: 10.1016/j.is.2007.07.001.

[12] Buiss, J. C. A. M.—van Dongen, B. F.—van der Aalst, W. M. P.: A Genetic Algorithm for Discovering Process Trees. IEEE Congress on Evolutionary Computation, 2012, 8 pp., doi: 10.1109/CEC.2012.6256458.

[13] Fahland, D. - VAN Der Aalst, W. M. P.: Model Repair - Aligning Process Models to Reality. Information Systems, Vol. 47, 2015, pp. 220-243, doi: 10.1016/j.is.2013.12.007.

[14] Polyvyanyy, A.- - van der Aalst, W. M. P.—-Ter Hofstede, A. H. M.Wynn, M. T.: Impact-Driven Process Model Repair. ACM Transactions on Software Engineering and Methodology, Vol. 25, 2016, No. 4, pp. 25-33, doi: 10.1145/2980764. 
[15] QI, H. D.-Du, Y. Y.-QI, L.-WAng, L.: An Approach to Repair Petri NetBased Process Models with Choice Structures. Enterprise Information Systems, Vol. 12, 2018, No. 8-9, pp. 1149-1179, doi: 10.1080/17517575.2018.1432768.

[16] Du, Y. Y.-Qi, L.-Zhou, M. C.: Analysis and Application of Logical Petri Nets to E-Commerce Systems. IEEE Transactions on Systems, Man, and Cybernetics: Systems, Vol. 44, 2014, No. 4, pp. 468-481, doi: 10.1109/TSMC.2013.2277696.

[17] Zhang, X. Z.-Du, Y. Y.-QI, L.-Sun, H. C.: An Approach for Repairing Process Models Based on Logic Petri Nets. IEEE Access, Vol. 6, 2018, pp. 29926-29939, doi: 10.1109/ACCESS.2018.2843137.

[18] Teng, Y.X.-Du, Y. Y.-QI, L.-Luan, W. J.: A Logic Petri Net-Based Method for Repairing Process Models with Concurrent Blocks. IEEE Access, Vol. 7, 2019, pp. 8266-8282, doi: 10.1109/ACCESS.2018.2890070.

[19] Adriansyah, A.: Aligning Observed and Modeled Behavior. Ph.D. Thesis, Technische Universiteit Eindhoven, 2014, pp. 139-149, doi: 10.6100/IR770080. 

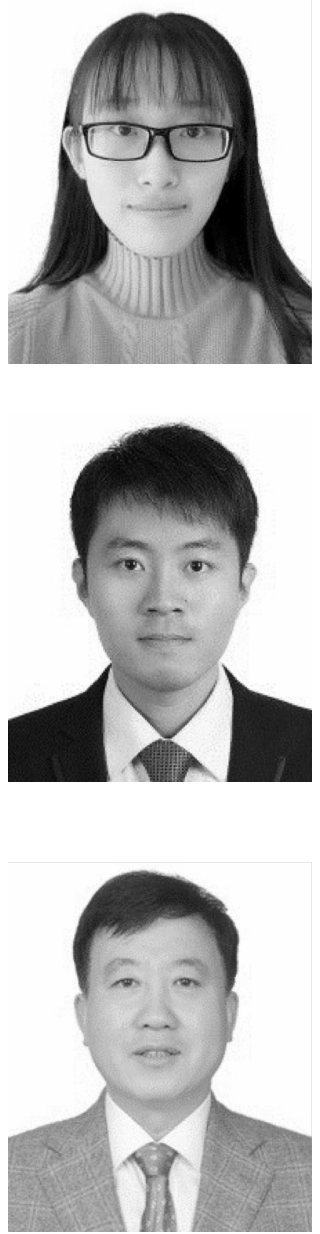

Yuanxiu Teng received her B.Sc. degree from Shandong University of Science and Technology, Qingdao, China, in 2017. She is now pursuing the M.Sc. degree in the College of Computer Science and Engineering, Shandong University of Science and Technology, Qingdao, China. Her current research interests are process mining, Petri nets and workflow.

Liang QI received his B.Sc. degree in information and computer science and M.Sc. degree in computer software and theory from Shandong University of Science and Technology, Qingdao, China, in 2009 and 2012, respectively, and the Ph.D. degree in computer software and theory from Tongji University, Shanghai, China, in 2017. He is currently Lecturer of computer science and technology at Shandong University of Science and Technology, Qingdao, China. His current research interests include Petri nets, discrete event systems, process mining and optimization algorithms.

Yuyue Du received his B.Sc. degree from Shandong University, Jinan, China, in 1982, the M.Sc. degree from Nanjing University of Aeronautics and Astronautics, Nanjing, China, in 1991, and the Ph.D. degree in computer application from Tongji University, Shanghai, China, in 2003. He is currently Professor at the College of Computer Science and Engineering, Shandong University of Science and Technology, Qingdao, China. His research interests are in formal engineering, Petri nets, real-time systems, process mining and workflows. 